TRANSACTIONS OF THE

AMERICAN MATHEMATICAL SOCIETY

Volume 364, Number 5, May 2012, Pages 2749-2781

S 0002-9947(2011)05518-2

Article electronically published on November 9, 2011

\title{
AN ALGEBRAIC CHAIN MODEL OF STRING TOPOLOGY
}

\author{
XIAOJUN CHEN
}

\begin{abstract}
A chain complex model for the free loop space of a connected, closed and oriented manifold is presented, and on its homology, the Gerstenhaber and Batalin-Vilkovisky algebra structures are defined and identified with the string topology structures. The gravity algebra on the equivariant homology of the free loop space is also modeled. The construction includes the non-simply connected case, and therefore gives an algebraic and chain level model of Chas-Sullivan's String Topology.
\end{abstract}

\section{INTRODUCTION}

This paper studies a chain complex model of the free loop space of a smooth, compact and oriented manifold. The purpose of our study is twofold: one is to give a down-to-earth algebraic model of the structures: the Gerstenhaber algebra, the Batalin-Vilkovisky algebra, and the gravity algebra of string topology discovered by Chas-Sullivan in [6] and 7] (see also Sullivan's survey [36), and the other is to relate these algebraic structures with some known ones, especially those from the Hochschild complexes of the cochain algebra of the manifold.

The fundamental but very subtle issue in string topology is the so-called open Frobenius algebra structure on the chain complex of a manifold. Given a smooth compact manifold $M$, geometrically the chain complex $C_{*}(M)$ forms a partially defined, differential graded (DG) open Frobenius algebra; namely, it is both a DG coalgebra under the diagonal approximation and a DG algebra under the transversal intersection, and the coproduct map is a map of bimodules:

$$
\Delta(\alpha \cap \beta)=\sum_{(\alpha)} \alpha^{\prime} \otimes \alpha^{\prime \prime} \cap \beta=\sum_{(\beta)} \alpha \cap \beta^{\prime} \otimes \beta^{\prime \prime},
$$

where $\Delta$ is the diagonal map: $\Delta \alpha=\sum_{(\alpha)} \alpha^{\prime} \otimes \alpha^{\prime \prime}, \Delta \beta=\sum_{(\beta)} \beta^{\prime} \otimes \beta^{\prime \prime}$. This identity is called the Frobenius or module compatibility. However, the Frobenius algebra is partially defined only when two chains are transversal. For example, a chain of dimension less than that of the manifold cannot intersect itself properly. While most of the string topology operations are based on the assumption of transversal chains, one might wonder if they can be defined on arbitrary chains and hence can be passed to the homology. More than that, it has been conjectured that the structures in string topology may not be a homotopy invariant of the manifold (see [36], Section 2.4 and also refer to [11]). To understand these problems, a chain

Received by the editors February 25, 2010 and, in revised form, October 16, 2010 and November 13, 2010.

2010 Mathematics Subject Classification. Primary 55P50, 55P35.

Key words and phrases. String topology, free loop space, Batalin-Vilkovisky algebra, Gerstenhaber algebra.

(C)2011 American Mathematical Society Reverts to public domain 28 years from publication 
level model of string topology cannot be avoided. This is the starting point of the current paper.

It turns out that a weak version of the Frobenius algebra, which we call the Frobenius-like algebra, on the Whitney forms of the manifold, is enough to model those operations in string topology. Giving $M$ a smooth cubulation (by cubulation we mean a decomposition of $M$ into cubes), recall that a Whitney polynomial form on $M$ is a differential form on $M$ with rational polynomial coefficients on each cube under the affine coordinates, with some obvious compatibilities. The set of Whitney polynomial forms, denoted by $A(M)$, forms a DG algebra, whose homology is the rational cohomology of $M$. Observe that by the compactness of $M$, the dual space of $A(M)$, the set of currents, denoted by $C(M)$, forms a complete DG coalgebra over $A(M)$. Moreover, $A(M)$ embeds into $C(M)$ as in the smooth differential forms case, which is in fact a quasi-isomorphism by Poincare duality. If we view $A(M)$ as a subcomplex of $C(M)$, and the wedge product on $A(M)$ as the intersection product (such a point of view is reasonable since on the homology level it does give the intersection product), then the induced coproduct on $A(M)$ :

$$
\Delta: A(M) \longrightarrow C(M) \hat{\otimes} C(M)
$$

satisfies the Frobenius compatibility (1.1) formally (by "formally" we mean the domain and range of $\Delta$ are in fact different). We would call the triple $(A(M), C(M), \iota)$, where $\iota$ is the embedding map, a DG open Frobenius-like algebra, whose homology exactly gives the Frobenius algebra on $H_{*}(M)$ in the usual sense.

Let $L M$ be the free loop space of $M . L M$ is a cosimplicial space (see Jones 221). The corresponding cosimplicial chain complex, denoted by $\widehat{C C}_{*}(C(M))$, is the complete cocyclic cobar complex of $C(M)$, and gives a chain complex model of $L M$ if $M$ is simply connected. Here $\widehat{C C}_{*}(C(M))$ is the coalgebra analogue of the cyclic bar complex and may also be viewed as the complete twisted tensor product of $C(M)$ with its complete cobar construction $\hat{\Omega}(C(M))$ with a twisted differential (see Section 3). We call the homology of $\widehat{C C}_{*}(C(M))$ the Hochschild homology of $C(M)$, and it is denoted by $H H_{*}(C(M))$. Furthermore, the embedding of $A(M)$ into $C(M)$ together with equation (1.1) guarantees that the linear map

$$
\iota \otimes i d: A(M) \hat{\otimes} \hat{\Omega}(C(M)) \longrightarrow C(M) \hat{\otimes} \hat{\Omega}(C(M))=\widehat{C C}_{*}(C(M))
$$

is a quasi-isomorphism of chain complexes (both with the twisted differential).

Both $A(M)$ and $\hat{\Omega}(C(M))$ are DG algebras; again by equation (1.1) one can check that the twisted product $A(M) \hat{\otimes} \hat{\Omega}(C(M))$ is a DG algebra under the twisted differential. The study of the commutativity of the induced product leads to the following:

Theorem 1.1 (Gerstenhaber algebra of the free loop space; cf. Theorem [5.7). Given a DG open Frobenius-like algebra $(A, C, \iota)$, its Hochschild homology $H_{*}(C)$ has the structure of a Gerstenhaber algebra. If the DG open Frobenius-like algebra comes from a simply connected closed manifold $M$, it is isomorphic to the Gerstenhaber algebra on the homology of the free loop space LM with rational coefficients, which coincides with the one of Chas-Sullivan in string topology.

The notion of Gerstenhaber algebras was first introduced by Gerstenhaber in his study of the deformation of associative algebras ([16]). The Gerstenhaber algebra of string topology is very similar to Gerstenhaber's original construction, and we 
will give a detailed comparison between them in Sections 5 and 6. Results similar to Theorem 1.1 have also been obtained by Félix-Thomas [14, Kaufmann [24] and Menichi [31. However, all their constructions require some finite dimensionality on the chain complex of a manifold: The arguments of [14] and [31] are based on rational homotopy theory, while Kaufmann directly works on the homology level (cf. [24, Section 5]). It is not easy to compare their constructions with the very geometric and pictorial construction of Chas-Sullivan. We hope our construction will build a bridge among them. On the other hand, once Theorem 1.1 is established, one may hope that all these constructions are isomorphic, because due to a result of Keller ([23, Sect. 4]), Gerstenhaber algebras on the Hochschild cohomology are preserved under quasi-isomorphic DG algebras.

Since the cocyclic cobar complex $\widehat{C C}_{*}(C(M))$ is the coalgebra analogue of the cyclic bar complex, we may introduce the coalgebra analogue of the Connes cyclic operator

$$
B: \widehat{C C}_{*}(C(M)) \longrightarrow \widehat{C C}_{*}(C(M)),
$$

which models the $S^{1}$-rotation on $L M$ on the chain level. From $A(M) \hat{\otimes} \hat{\Omega}(C(M)) \simeq$ $\widehat{C C}_{*}(C(M))$ we may pull back $B$ to the homology of $A \hat{\otimes} \hat{\Omega}(C(M))$ and obtain the following (also obtained in [14, 24] and [31] by the authors cited above):

Theorem 1.2 (Batalin-Vilkovisky algebra of the free loop space; cf. Theorem [7.4). The functor in Theorem 1.1 is in fact a functor to the category of Batalin-Vilkovisky algebras, which is isomorphic to the Batalin-Vilkovisky algebra of Chas-Sullivan in string topology in the case of simply connected manifolds.

The Batalin-Vilkovisky algebras are highly related to topological field theories; see Getzler [17. In fact, Getzler showed that a 2-dimensional (genus zero) topological conformal field theory (TCFT) contains a natural Batalin-Vilkovisky structure. Later in [18, he continued to show that the equivariant TCFT (again in the genus zero case) has a structure of a gravity algebra, which may be viewed as a generalized Lie algebra. By considering the cyclic homology of the DG coalgebra $C(M)$, we obtain:

Theorem 1.3 (Gravity algebra of the free loop space; cf. Theorem 8.5). Given a $D G$ open Frobenius-like algebra $(A, C, \iota)$, its cyclic homology has the structure of a gravity algebra, which is isomorphic to the gravity algebra of Chas-Sullivan on the equivariant homology of the free loop space.

An operadic interpretation of the gravity algebra in string topology has been obtained by Westerland [41, Section 7]. The Lie algebra part in the above theorem has previously been studied by Menichi in [30] and 31, where he chooses a different DG algebra for $M$.

There is an extensive literature on string topology. Thomas Tradler, in his Ph.D. thesis [37, first identified the loop homology (the homology of $L M$ with a degree shifting) with the Hochschild cohomology of the cochain complex of the manifold as Gerstenhaber algebras. His construction uses the singular chain complex of the manifold. At the same time, in the beautiful paper of Cohen-Jones [10, the authors gave a homotopy-theoretic realization of string topology via the Thom-Pontrjagin construction, and also showed the isomorphism of the Hochschild cohomology with the loop homology. Similar results have also been obtained by Merkulov ([32]) via 
K.-T. Chen's iterated path integrals, and by Félix-Thomas-Vigué-Poirrier (15]) via Sullivan's minimal model theory.

As for the Gerstenhaber and Batalin-Vilkovisky algebras, besides the works of Félix-Thomas, Kaufmann and Menichi, Voronov [39] showed that the loop homology is an algebra over the framed "cactus" operad, while the latter is homotopic to the framed little disk operad, which then implies the Batalin-Vilkovisky algebra on the loop homology as well. For more details of their results, see also CohenHess-Voronov 9]. McClure, in his paper 29, constructed a chain-level intersection theory by using the PL chains (the intersection is partially defined), and from this he was able to show the Gerstenhaber and Batalin-Vilkovisky algebras on their cocyclic cobar complex. In our construction of the Frobenius-like algebra, the set of currents is the chain complex of the manifold, and on its subcomplex, the Whitney forms, the intersection product is defined. Therefore, in some sense, the present paper may be viewed as a sequel to Tradler and McClure's work.

At last we remark that, according to the point of view of Dennis Sullivan, to correctly model the Frobenius structure of the chain complex of a manifold, one can: 1) either make the Frobenius compatibility equation (1.1) strictly hold but with the price that the intersection product is partially defined; 2) or diffuse the chains on the manifold such that the intersection product is fully defined but with the price that equation (1.1) only holds up to homotopy. While the method applied in this paper takes the first point of view, the second point of view is also applicable: in the paper [21, Hamilton and Lazarev constructed a cyclic $\mathrm{Com}_{\infty}$ algebra which models the chain complex of a manifold, and then the result of Costello ([13]) shows that the Hochschild cohomology of the cyclic $\mathrm{Com}_{\infty}$ algebras, which is isomorphic to the loop homology, naturally endows the structures of the Batalin-Vilkovisky algebra. Recently McClure and Wilson (in a private communication) have constructed a homotopy open Frobenius algebra on the chain complex of a manifold. The application of their construction to model string topology, especially to obtain the higher genus string topology operations on the equivariant homology of the free loop space, will appear elsewhere. A Thom-Pontrjagin method of these higher genus operations on the homology space, has been obtained by V. Godin ([20]).

The rest of the paper is devoted to the proof of the above theorems. In Section 2, we study the Frobenius algebra structure on the chain complex of a smooth manifold. In Section 3, we construct a chain complex model for the free loop space of a simply connected manifold. In Sections 4-6, we study the Gerstenhaber algebra structure on its free loop space, from our point of view. In Section 7, we define and identify the Batalin-Vilkovisky algebra structure on the free loop space. In Section 8 , we recall a model for the equivariant chain complex of the free loop space and identify the gravity algebra on its homology. In the last section, we sketch the constructions of the above structures on nonsimply connected manifolds.

\section{The Chain COMPleX of A MANiFold}

Let $M$ be a smooth, closed oriented manifold. The rational cohomology of $M$, $H^{*}(M ; \mathbb{Q})$, has the following structure: 1$)$ it is a graded commutative algebra; 2) there is a non-degenerate pairing on it by Poincaré duality. We usually call a linear space which satisfies 1) and 2) a closed Frobenius algebra. Alternatively, a closed Frobenius algebra is a linear space $V$ which is a graded commutative algebra and a graded cocommutative coalgebra with both unit and counit, and moreover, 
the coproduct is a map of bimodules:

$$
\Delta(\alpha \cdot \beta)=\sum_{(\alpha)} \alpha^{\prime} \otimes \alpha^{\prime \prime} \cdot \beta=\sum_{(\beta)} \alpha \cdot \beta^{\prime} \otimes \beta^{\prime \prime} .
$$

By the isomorphism $H^{*}(M ; \mathbb{Q}) \cong H_{n+*}(M ; \mathbb{Q})$ (in this paper we grade the cohomology negatively, and correspondingly the differential has degree -1$)$, the above statement says that $H_{*}(M ; \mathbb{Q})$ with the intersection product and diagonal coproduct forms a closed Frobenius algebra. However, such an algebraic structure does not hold on the chain level, since the intersection of two chains is defined only when they are transversal.

Definition 2.1 (Whitney polynomial differential forms). Let $M$ be a cubulated topological space. A cubical Whitney polynomial differential form $x$ on $M$ is a collection of differential forms, one on each cube, such that:

(1) the coefficients of these forms on each cube are polynomials over $\mathbb{Q}$ with respect to the affine coordinates of the cubes;

(2) they are compatible under restriction to faces; i.e., if $\tau$ is face of $\sigma$, then $x_{\sigma} \mid \tau=x_{\tau}$.

The set of Whitney polynomial forms on $M$ is denoted by $A(M)$.

A smooth manifold $M$ always admits a smooth cubulation. For example, one construction of a smooth cubulation is as follows: by the famous theorem of Whitehead [42, any smooth manifold admits a smooth triangulation, and then the dual decomposition of such a triangulation naturally gives a smooth cubulation of $M$. In the following we will repeatedly be using the products of manifolds, and cubulated spaces have an advantage that the products of cubulated spaces are naturally cubulated, while triangulated spaces do not have this property. In the following we fix a smooth cubulation for $M$.

Since $M$ is closed, the cubes on $M$ are finite in number, and therefore if we denote by $A^{p}(M)$ the set of Whitney forms of grading less than or equal to $p$ (here by grading we mean the sum of the degree of the form and the degree of the polynomial coefficient), then

$$
A^{0}(M) \subset A^{1}(M) \subset \cdots, \quad \operatorname{dim} A^{p}(M)<\infty \text { for } p=0,1, \cdots
$$

and $A(M)=\underline{\lim } A^{p}(M)$. Moreover $A(M)$ has a unit and augmentation which are given by the constant functions $A^{0}(M) \cong \mathbb{Q}$, where by augmentation we mean an algebra map from $A(M)$ to $\mathbb{Q}$.

Proposition 2.2. Let $A(M)$ be the Whitney polynomial forms of $M$. Then:

(1) $A(M)$, under wedge $\wedge$ and exterior differential d, forms a commutative $D G$ algebra.

(2) The Whitney forms may be mapped to the cubical cochains of the space as follows:

$$
\begin{aligned}
\rho: A(M) & \longrightarrow C^{*}(M ; \mathbb{Q}), \\
x & \longmapsto\left\{I^{n} \mapsto \int_{I^{n}} x\right\}, \text { for any } I^{n},
\end{aligned}
$$

which is a chain map. 
Proposition 2.2 (1) holds because $\wedge$ and $d$ are both natural under restriction to faces, and (2) follows from Stokes' theorem. Moreover, $A(M)$ computes the cohomology of $M$.

Theorem 2.3 (de Rham's theorem for Whitney forms). Let $M$ be a cubulated topological space. Then $\rho$ is a chain equivalence of DG algebras, i.e.

$$
\rho^{*}: H^{*}(A(M), d) \underset{\text { alg }}{\stackrel{\cong}{\longrightarrow}} H^{*}(M ; \mathbb{Q}) .
$$

Proof. See Cenkl-Porter [5, Theorem 4.1.

We next show that the dual space of the Whitney forms forms a complete DG coalgebra. First, recall that, if $C=\lim C_{p}$ and $D=\lim _{\longleftarrow} D_{q}$ are two inverse limit systems of filtered $k$-vector spaces, then the complete tensor product of $C$ and $D$, denoted by $C \hat{\otimes} D$, is given by

$$
C \hat{\otimes} D:=\lim _{\longleftarrow} \bigoplus_{r=p+q} C_{p} \otimes D_{q} .
$$

Definition 2.4 (Complete coalgebra). Suppose $C=\lim _{\longleftarrow} C_{p}$ is an inverse system. A complete coalgebra on $C$ is $C$ together with a complete diagonal

$$
\hat{\Delta}: C \rightarrow C \hat{\otimes} C,
$$

which is a map of complete spaces and which is coassociative. The counit and coaugmentation of $C$ are non-zero coalgebra maps $C \rightarrow k$ and $k \rightarrow C$. A complete DG coalgebra is $C$ together with a differential which commutes with the complete diagonal map.

Lemma 2.5. Let $M$ be a smooth manifold and $A(M)$ be the Whitney polynomial forms of $M$. Let $C(M):=\operatorname{Hom}(A(M), \mathbb{Q})$ be the space of currents; then $C(M)$ forms a differential graded complete coalgebra with a counit and a coaugmentation.

Proof. Note that

$$
C(M)=\operatorname{Hom}(A(M), \mathbb{Q})=\operatorname{Hom}\left(\underline{\lim } A^{p}(M), \mathbb{Q}\right)=\underset{\longleftarrow}{\lim } \operatorname{Hom}\left(A^{p}(M), \mathbb{Q}\right)
$$

and that the wedge product on $A(M)$ respects the filtration (2.2), that is, $\wedge$ : $A(M) \otimes A(M) \rightarrow A(M)$ induces a DG map

$$
\begin{aligned}
\Delta: C(M)=\operatorname{Hom}(A(M), \mathbb{Q}) \longrightarrow & \operatorname{Hom}(A(M) \otimes A(M), \mathbb{Q}) \\
& =\operatorname{Hom}\left(\underset{\lim }{\longrightarrow} A^{p}(M) \otimes \underline{\lim } A^{p}(M), \mathbb{Q}\right) \\
& =\operatorname{Hom}\left(\stackrel{\lim }{\longrightarrow}_{r=p+q} A^{p}(M) \otimes A^{q}(M), \mathbb{Q}\right) \\
& =\lim _{r=p+q} \operatorname{Hom}\left(A^{p}(M), \mathbb{Q}\right) \otimes \operatorname{Hom}\left(A^{q}(M), \mathbb{Q}\right) \\
& =C(M) \hat{\otimes} C(M) .
\end{aligned}
$$

The counit and coaugmentation come from the unit and augmentation of $A(M)$.

The above lemma is the key observation in reconstructing the string topology operations; it allows us to reduce all the computations involving the cochain complex of a manifold to the limit of finite-dimensional objects. Since $A(M)$ computes the 
rational cohomology of $M$, by the Universal Coefficient Theorem, $C(M)$ computes the rational homology of $M$. We call $(C(M), \Delta, d)$ the complete DG coalgebra of $M$. As in the smooth case, the Whitney forms embed into the currents, which is a quasi-isomorphism by Poincaré duality:

Proposition 2.6. The embedding of $A(M)$ into $C(M)$, given by

$$
\iota: A(M) \longrightarrow C(M): x \longmapsto\left\{y \mapsto \int_{M} x \wedge y\right\}
$$

is a quasi-isomorphism of chain complexes.

Note that the dual space of a commutative algebra is always a bimodule of that algebra via the left and right premultiplication. We have that $C(M)$ is a DG $A(M)$-bimodule, so if we denote $\Delta(\iota x)$ by $\Delta x$, for any $x \in A(M)$, then:

Proposition 2.7. For any $x, y \in A(M)$,

$$
\Delta(x y)=\sum_{(x)} x^{\prime} \hat{\otimes} x^{\prime \prime} y=\sum_{(y)} x y^{\prime} \hat{\otimes} y^{\prime \prime} .
$$

The proof follows from a direct check. However, one should note that the sum in equation (2.4) may be infinite. Equation (2.4) is much like equation (1.1), with $\Delta: A(M) \rightarrow C(M) \hat{\otimes} C(M)$ instead of $\Delta: C(M) \rightarrow C(M) \hat{\otimes} C(M)$. This shows that although we may not be able to define the intersection and coproduct simultaneously on the chain complex $C_{*}(M)$, the pair $(A(M), C(M))$ is good enough that we may define the intersection on $A(M)$ and the coproduct on $C(M)$ while the Frobenius identity still holds.

Moreover, the coproduct of $A(M)$ factors through $A(M) \hat{\otimes} C(M)$; namely, if we denote a basis of $A(M)$ by $\left\{y_{i}\right\}$, and let $\left\{y_{i}^{*}\right\}$ be the dual basis, then

$$
\Delta x=\sum_{i} \iota\left(x y_{i}\right) \hat{\otimes} y_{i}^{*} .
$$

Hence we may formally write

$$
\Delta x=\sum_{i} x y_{i} \hat{\otimes} y_{i}^{*}, \quad \text { for all } x \in A(M) .
$$

Let us summarize the above observations:

(1) $A(M)$ is a DG commutative algebra;

(2) $C(M)$ is a (complete) DG cocommutative coalgebra over $A(M)$;

(3) there is a quasi-isomorphic embedding of $A(M)$-modules $\iota: A(M) \rightarrow C(M)$ which makes $A(M)$ a (complete) DG bi-comodule over $C(M)$.

Definition 2.8. We call a triple $(A, C, \iota)$ which satisfies the above conditions (1), (2) and (3) a DG open Frobenius-like algebra.

The homology of $(A, C, \iota)$ is defined to be the homology of $A$ or $C$, which forms a Frobenius algebra naturally. In the case of Whitney forms and currents on a manifold, this gives exactly the closed Frobenius algebra structure on $H_{*}(M ; \mathbb{Q})$. 


\section{Chain COMplex MOdel of The Free loop SPACE}

In this section we recall some facts on the cosimplicial chain complex model of the free loop space. The idea is due to K.-T. Chen [8] and Jones [22] (see also Getzler-Jones-Petrack [19]).

Let $\Delta^{n}=\left\{\left(t_{1}, t_{2}, \cdots, t_{n}\right) \in \mathbb{R}^{n}: 0 \leq t_{1} \leq t_{2} \leq \cdots \leq t_{n} \leq 1\right\}$ be the standard $n$-simplex in $\mathbb{R}^{n}$. For each $n$, we have the evaluation map

$$
\Psi_{n}: L M \times \Delta^{n} \longrightarrow \underbrace{M \times M \times \cdots \times M}_{n+1},
$$

which is given by $\Psi_{n}\left(\gamma,\left(t_{1}, t_{2}, \cdots, t_{n}\right)\right)=\left(\gamma(0), \gamma\left(t_{1}\right), \cdots, \gamma\left(t_{n}\right)\right)$. By the chain equivalence of $C_{*}\left(M^{\times n+1}\right)$ with $C_{*}(M)^{\otimes n+1}$, consider the composition

$$
C_{*}(L M) \longrightarrow C_{*}(L M) \otimes\left[\Delta^{n}\right] \stackrel{\Psi_{n \#}}{\longrightarrow} C_{*}(M)^{\otimes n+1},
$$

where $\left[\Delta^{n}\right]$ is the fundamental chain of $\Delta^{n}, \Psi_{n \#}$ is the chain map induced by $\Psi_{n}$, and we obtain a chain model for $L M$. Before doing that, let us introduce the cocyclic cobar complex of a DG coalgebra, which is the coalgebra analogue of the cyclic bar complex: Let $(C, d)$ be a DG cocommutative coalgebra; the cocyclic cobar complex $C C_{*}(C)$ of $C$ is the direct product

$$
\prod_{n=0}^{\infty} C \otimes(\Sigma C)^{\otimes n}
$$

where $\Sigma$ is the desuspension functor of $C$ (the functor which simply shifts the degree of $C$ down by 1 ), with differential defined by

$$
\begin{aligned}
& b\left(a \otimes\left[a_{1}|\cdots| a_{n}\right]\right) \\
& :=d a \otimes\left[a_{1}|\cdots| a_{n}\right]-\sum_{i}(-1)^{|a|+\left|\left[a_{1}|\cdots| a_{i-1}\right]\right|} a \otimes\left[a_{1}|\cdots| d a_{i}|\cdots| a_{n}\right] \\
& -\sum_{i} \sum_{\left(a_{i}\right)}(-1)^{|a|+\left|\left[a_{1}|\cdots| a_{i-1} \mid a_{i}^{\prime}\right]\right|} a \otimes\left[a_{1}|\cdots| a_{i}^{\prime}\left|a_{i}^{\prime \prime}\right| \cdots \mid a_{n}\right] \\
& +\sum_{(a)}(-1)^{\left|a^{\prime}\right|} a^{\prime} \otimes\left(\left[a^{\prime \prime}\left|a_{1}\right| \cdots \mid a_{n}\right]-(-1)^{\left(\left|a^{\prime \prime}\right|-1\right)\left|\left[a_{1}|\cdots| a_{n}\right]\right|}\left[a_{1}|\cdots| a_{n} \mid a^{\prime \prime}\right]\right) .
\end{aligned}
$$

Here we adopt the usual convention by writing elements in $C \otimes(\Sigma C)^{\otimes n}$ in the form $a \otimes\left[a_{1}|\cdots| a_{n}\right]$. That $b^{2}=0$ follows from the coassociativity of the coproduct of $C$. Note that the dual complex of a DG coalgebra is a DG algebra; it is easy to see that the dual complex of $C C_{*}(C)$ is the completion of the cyclic bar complex (also called the Hochschild complex) of the dual DG algebra of $C$, where the completion is taken with respect to the number of components. We will call the homology of $C C_{*}(C)$ the Hochschild homology of the coalgebra $C$, denoted by $H H_{*}(C)$. For an elegant treatment of the cyclic bar complex of a DG algebra, see, for example, [19] and Loday [25].

If, moreover, $C$ is counital and coaugmented, we may consider the reduced cocyclic cobar complex of $C$, which is obtained from $C C_{*}(C)$ by identifying elements $x \otimes\left[a_{1}|\cdots| 1|\cdots| a_{n}\right]$ with zero, where 1 is the image of the coaugmentation. To distinguish them, we always write the latter as $\prod_{n} C \otimes(\Sigma \bar{C})^{\otimes n}$. In the following when mentioning the cocyclic cobar complex we shall always assume it is reduced, since in our construction of the DG coalgebra of a manifold $M, C(M)$ is always counital and coaugmented. 
We may extend the above definition to the case of complete DG coalgebras, which is given by

$$
\widehat{C C}_{*}(C):=\prod_{n=0}^{\infty} C \hat{\otimes}(\Sigma \bar{C})^{\hat{\otimes} n},
$$

with the differential $b$ extending to the complete tensor product.

Theorem 3.1. Let $M$ be a simply connected manifold, and let $C(M)$ (written $C$ for short) be the complete DG coalgebra of $M$. There is a chain equivalence

$$
\phi:\left(C_{*}(L M), \partial\right) \longrightarrow\left(\widehat{C C}_{*}(C), b\right) .
$$

Proof. The chain map is induced from

$$
\begin{aligned}
\phi: C_{*}(L M) & \longrightarrow \prod_{n=0}^{\infty} C \hat{\otimes}(\Sigma \bar{C})^{\hat{\otimes} n}, \\
\alpha & \longmapsto \sum \Phi_{n \#}\left(\alpha \otimes\left[\Delta^{n}\right]\right),
\end{aligned}
$$

where $\Phi_{n \#}$ is the composition of $\Psi_{n \#}$ with the embedding of $C_{*}(M)$ into $C(M)$. Note that $\phi$ is a chain map: the differential of any element in $\prod C \hat{\otimes}(\Sigma \bar{C})^{\hat{\otimes} n}$ contains two parts: one is those terms containing the differential of the elements in $C$, the other is those terms that involve the coproduct of the elements in $C$. If we write $b(\alpha)=b^{\mathrm{I}}(\alpha)+b^{\mathrm{II}}(\alpha)$ referring to these two parts, namely, $b^{\mathrm{I}}(\alpha)=(3.2)$ and $b^{\mathrm{II}}(\alpha)=(3.3)+(3.4)$, then

$$
\begin{aligned}
\phi(\partial \alpha) & =\sum \Phi_{n \#}\left(\partial \alpha \otimes\left[\Delta^{n}\right]\right) \\
& =\sum \Phi_{n \#}\left(\partial\left(\alpha \otimes\left[\Delta^{n}\right]\right)-(-1)^{|\alpha|} \alpha \otimes \partial\left[\Delta^{n}\right]\right) \\
& =\partial \circ\left(\sum \Phi_{n \#}\left(\alpha \otimes\left[\Delta^{n}\right]\right)\right)-\sum \Phi_{n \#}\left(\sum_{i}(-1)^{|\alpha|+i} \alpha \otimes \delta_{i}\left[\Delta^{n-1}\right]\right) \\
& =b^{\mathrm{I}} \circ \phi(\alpha)+b^{\mathrm{II}} \circ \phi(\alpha)=b \circ \phi(\alpha),
\end{aligned}
$$

where in the above, $\delta_{i}$ is the identification of $\Delta^{n-1}$ with the $i$-th face of $\Delta^{n}$. More precisely, the last equality holds due to the following: Define two groups of maps $\left\{\delta_{i}: \Delta^{n-1} \rightarrow \Delta^{n}\right\}$ by

$$
\begin{aligned}
\delta_{0}\left(t_{1}, \cdots, t_{n-1}\right) & =\left(0, t_{1}, \cdots, t_{n-1}\right), \\
\delta_{i}\left(t_{1}, \cdots, t_{n-1}\right) & =\left(t_{1}, \cdots, t_{i}, t_{i}, \cdots, t_{n-1}\right), \quad 1 \leq i \leq n-1, \\
\delta_{n}\left(t_{1}, \cdots, t_{n-1}\right) & =\left(t_{1}, \cdots, t_{n-1}, 1\right),
\end{aligned}
$$

and $\left\{\delta_{i}: M^{\times n} \rightarrow M^{\times n+1}\right\}$ by

$$
\begin{aligned}
\delta_{0}\left(x_{0}, \cdots, x_{n-1}\right) & =\left(x_{0}, x_{0}, \cdots, x_{n-1}\right), \\
\delta_{i}\left(x_{0}, \cdots, x_{n-1}\right) & =\left(x_{0}, \cdots, x_{i}, x_{i}, \cdots, x_{n-1}\right), \quad 1 \leq i \leq n-1, \\
\delta_{n}\left(x_{0}, \cdots, x_{n-1}\right) & =\left(x_{0}, \cdots, x_{n-1}, x_{0}\right) .
\end{aligned}
$$

Then the following diagram commutes:

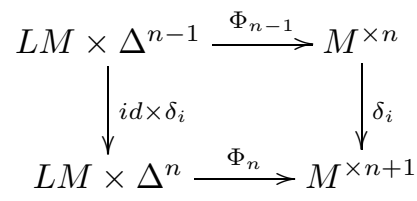


for all $0 \leq i \leq n$. Therefore,

$$
\Phi_{n \#}\left(\sum_{i}(-1)^{i} \alpha \otimes \delta_{i}\left[\Delta^{n-1}\right]\right)=\sum_{i}(-1)^{i} \delta_{i \#} \circ \Phi_{n-1 \#}\left(\alpha \otimes\left[\Delta^{n-1}\right]\right),
$$

and if we shift the degree of the last $n-1$ components in $C_{*}(M)^{\otimes n}$ down by one (which then multiplies $(-1)^{i}$ to the image of $\delta_{i \#} \circ \Phi_{n-1 \#}$, hence the $(-1)^{i}$ cancel) and sum over all $n \geq 0$, we obtain

$$
\sum_{n=0}^{\infty} \Phi_{n \#}\left(\sum_{i}(-1)^{|\alpha|+i} \alpha \otimes \delta_{i}\left[\Delta^{n-1}\right]\right)=b^{\mathrm{II}} \circ \phi(\alpha) .
$$

The rest of the proof follows from a spectral sequence argument; see, for example, Bousfield [3], §4.1 or Rector [34, Corollary 5.2.

In the original construction of K.-T. Chen and Jones, both authors used the cochain algebra of the manifold. In our construction, the homology of the complete DG coalgebra $C(M)$ is a graded coalgebra (not in the complete sense); and therefore, the subtlety of using the complete DG coalgebra $C(M)$ seems to disappear: one deduces that, by the comparison of spectral sequences, $\prod_{n} C_{*}(M) \otimes \Sigma C_{*}(M)^{\otimes n}$ and $\prod_{n} C(M) \hat{\otimes} \Sigma C(M)^{\hat{\otimes} n}$ are quasi-isomorphic provided that $M$ is simply connected.

We can also describe the $S^{1}$-action on $L M$ in the above chain complex model, given by the coalgebra version of Connes' cyclic operator (cf. Connes [12] and Jones [22]):

$$
\begin{aligned}
B: \widehat{C C}_{*}(C) & \longrightarrow \widehat{C C}_{*}(C), \\
a \otimes\left[a_{1}|\cdots| a_{n}\right] \longmapsto \sum_{i}(-1)^{\left|\left[a_{i}|\cdots| a_{n}\right]\right|\left|\left[a_{1}|\cdots| a_{i-1}\right]\right|} \varepsilon(a) a_{i} & \\
& \otimes\left[a_{i+1}|\cdots| a_{n}\left|a_{1}\right| \cdots \mid a_{i-1}\right],
\end{aligned}
$$

where $\varepsilon$ is the counit. One can easily check that $B^{2}=0$ and $b B+B b=0$.

Theorem 3.2. Let $M$ be a simply connected manifold. Let

$$
J: C_{*}(L M) \longrightarrow C_{*+1}(L M)
$$

be the degree-one map given by the composition

$$
\begin{aligned}
& L M \quad \longrightarrow L M \times S^{1} \stackrel{A}{\longrightarrow} L M, \\
& \alpha \longmapsto \alpha \otimes\left[S^{1}\right] \quad \longmapsto A_{\#}\left(\alpha \otimes\left[S^{1}\right]\right),
\end{aligned}
$$

where $A$ is the rotation: $A(f, s)=f(s+\cdot)$, for any $f \in L M$ and $s \in S^{1}$, and $\left[S^{1}\right]$ is the fundamental cycle of $S^{1}$. We have the following chain equivalence:

$$
\left(C_{*}(L M), d, J\right) \stackrel{\simeq}{\longrightarrow}\left(\widehat{C C}_{*}(C), b, B\right) .
$$

Proof. Decompose $S^{1} \times \Delta^{n}$ into $n+1$ standard $(n+1)$-simplices: $S^{1} \times \Delta^{n}=$ $\bigcup_{i=1}^{n+1} \Delta_{i}^{n+1}$, where

$$
\begin{aligned}
\Delta_{i}^{n+1} & :=\left\{0 \leq s \leq \cdots \leq s+t_{i-1} \leq 1 \leq s+t_{i} \leq \cdots \leq s+t_{n} \leq 2\right\} \\
& =\left\{0 \leq s+t_{i}-1 \leq \cdots \leq s+t_{n}-1 \leq s \leq \cdots \leq s+t_{i-1} \leq 1\right\},
\end{aligned}
$$


and let $r_{i}$ be the inclusion of $\Delta_{i}^{n+1}$ into $S^{1} \times \Delta^{n}$. Then the following diagram commutes:

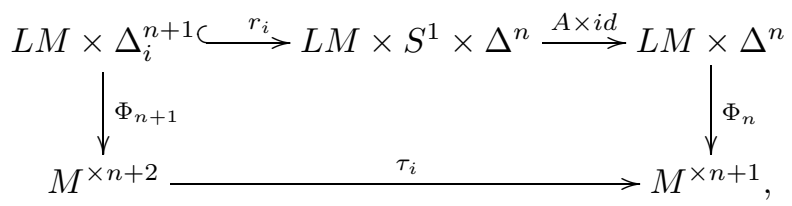

where $\tau_{i}: M^{\times n+2} \rightarrow M^{\times n+1}$ is given by

$$
\tau_{i}\left(x_{0}, x_{1}, \cdots, x_{n+1}\right)=\left(x_{n-i+1}, \cdots, x_{n+1}, x_{1}, \cdots, x_{n-i}\right),
$$

and $\Phi_{n+1}$ is evaluated at $\left(f,\left(0, s+t_{i}-1, \cdots, s+t_{n}-1, s, \cdots, s+t_{i-1}\right)\right)$. Applying the chain functor we obtain:

$$
\begin{aligned}
\Phi_{n \#}\left(J \alpha \otimes\left[\Delta^{n}\right]\right) & =\Phi_{n \#}\left(A_{\#}\left(\alpha \otimes\left[S^{1}\right]\right) \otimes\left[\Delta^{n}\right]\right) \\
& =\Phi_{n \#} \circ(A \times i d)_{\#}\left(\alpha \otimes\left[S^{1}\right] \otimes\left[\Delta^{n}\right]\right) \\
& =\Phi_{n \#} \circ(A \times i d)_{\#}\left(\sum_{i=1}^{n+1}\left(i d \otimes r_{i}\right)\left(\alpha \otimes\left[\Delta_{i}^{n+1}\right]\right)\right) \\
& =\sum_{i=1}^{n+1} \tau_{i \#} \circ \Phi_{n+1 \#}\left(\alpha \otimes\left[\Delta_{i}^{n+1}\right]\right) .
\end{aligned}
$$

In particular, if $\Phi_{n+1 \#}\left(\alpha \otimes\left[\Delta^{n+1}\right]\right)=a \otimes\left[a_{1}|\cdots| a_{n+1}\right]$, then $\tau_{i \#} \circ \Phi_{n+1 \#}\left(\alpha \otimes\left[\Delta_{i}^{n+1}\right]\right)= \begin{cases}0, & \text { if }|a| \neq 0 \\ \pm \varepsilon(a) a_{i} \otimes\left[a_{i+1}|\cdots| a_{n+1}\left|a_{1}\right| \cdots \mid a_{i-1}\right], & \text { otherwise }\end{cases}$ where in the $|a| \neq 0$ case the value is zero because it is a degenerate chain (the degrees of the two sides are not equal while $\tau_{i \#}$ is a chain map), and therefore

$$
\begin{aligned}
\Phi_{n \#}\left(J \alpha \otimes\left[\Delta^{n}\right]\right) & =\sum_{i=1}^{n+1} \tau_{i \#} \circ \Phi_{n+1 \#}\left(\alpha \otimes\left[\Delta_{i}^{n+1}\right]\right) \\
& =B \circ \Phi_{n+1 \#}\left(\alpha \otimes\left[\Delta^{n+1}\right]\right)
\end{aligned}
$$

by definition. Summing over all $n \geq 0$, we obtain (3.5) as claimed.

In the above definition of $\widehat{C C}_{*}(C)$, if we write

$$
\hat{\Omega}(C):=\prod_{n=0}^{\infty}(\Sigma \bar{C})^{\hat{\otimes} n},
$$

which is the complete cobar construction of $C$, then

$$
\widehat{C C}_{*}(C)=C \hat{\otimes} \hat{\Omega}(C) .
$$

This has an interpretation of Brown's twisted tensor product theory [4]: For the fibration

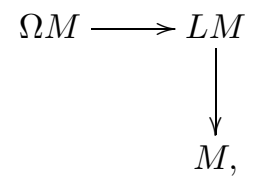

the theorem of Brown says that there is a chain equivalence between the chain complex of the total space $L M$ and the "twisted" tensor product of the chain 
complexes of the base $M$ and the fiber $\Omega M$. Since such a point of view plays a role in understanding the Chas-Sullivan loop product on the loop homology, let us describe this in more detail.

Definition 3.3 (Twisting cochain). Let $(C, d)$ be a DG coalgebra over a field $k$ with coaugmentation $\eta: k \rightarrow C$, and let $(A, \delta)$ be a DG algebra. A twisting cochain is a degree -1 linear map $\Phi=\sum_{q} \Phi_{q}: C_{q} \rightarrow A_{q-1}$ such that

(1) $\Phi_{0}\left(1_{C}\right)=0$, where $1_{C}$ is the image of 1 under $\eta$;

(2) $\delta \circ \Phi_{q}=-\Phi_{q-1} \circ d-\sum_{k}(-1)^{k} \Phi_{k} \cup \Phi_{q-k} \circ \Delta$.

Let $(M, p)$ be a connected pointed topological space, and let $S_{*}(M)$ be the 1reduced singular chain complex of $M$ (here by "1-reduced singular chain complex" we mean the singular chains generated by simplexes taking the vertices of the standard simplex into the basepoint $p$ ). The Alexander-Whitney diagonal approximation gives a DG coassociative coalgebra on $S_{*}(M)$. Now let $C_{*}(\Omega M)$ be the chain complex of the based loop space of $M$ at the base point $p$. Brown constructs a twisting cochain $\Phi: S_{*}(M) \rightarrow C_{*-1}(\Omega M)$, which, roughly speaking, fills each simplex with paths connecting its first and last vertices. Such a construction is similar to the one of Adams [1] (with a minor modification). In fact, Adams proved that if $M$ is simply connected, then the cobar construction of $S_{*}(M)$ is chain equivalent to $C_{*}(\Omega M)$.

Now let $F \rightarrow E \stackrel{\pi}{\rightarrow}(M, p)$ be a Hurewicz fibration with fiber $F=\pi^{-1}(p)$. Taking any loop $\gamma \in \Omega_{p} M$, for any point $f \in F$ we may lift $\gamma$ to $E$ ending at $f$. Denoting the initial point of the path to be $\gamma f$, we get a continuous action of $\Omega_{p} M$ on $F$, which induces a DGA action on the chain level:

$$
\circ: C_{*}(\Omega M) \otimes C_{*}(F) \longrightarrow C_{*}(F) .
$$

Define an operator $\partial_{\Phi}$ on $S_{*}(M) \otimes C_{*}(F)$ as follows:

$$
\partial_{\Phi}(a \otimes f):=\partial a \otimes f+(-1)^{|a|} a \otimes \partial f+\sum(-1)^{\left|a^{\prime}\right|} a^{\prime} \otimes \Phi\left(a^{\prime \prime}\right) \circ f .
$$

Then $\partial_{\Phi}^{2}=0$. We call $\partial_{\Phi}$ the twisted differential and $\left(S_{*}(M) \otimes C_{*}(F), \partial_{\Phi}\right)$ the twisted tensor product. The theorem of Brown is that, for the fiber bundle $F \rightarrow E \rightarrow M$, there is a chain equivalence

$$
\phi:\left(S_{*}(M) \otimes C_{*}(F), \partial_{\Phi}\right) \longrightarrow\left(C_{*}(E), \partial\right) .
$$

Now for the free loop space of a manifold $L M$, the fibration $\Omega_{p} M \rightarrow L M \rightarrow$ $(M, p)$ has a natural lifting function, which is given as follows: for any $\gamma:[0,1] \rightarrow$ $M, \gamma(0)=q, \gamma(1)=p$, then

$$
\begin{aligned}
\gamma: \Omega_{p} M & \longrightarrow \Omega_{q} M, \\
x & \longmapsto \gamma x \gamma^{-1} .
\end{aligned}
$$

When $p=q$, the action is exactly the (left) adjoint action of $\Omega_{p} M$ on itself. Passing to the chain level, it gives the (left) adjoint action of the Hopf algebra $C_{*}(\Omega M)$ on itself. Such an observation was also obtained by McCleary [27.

By the result of Adams [1, if $C$ is the DG coalgebra of $M$, then the cobar construction $\Omega(C)$ gives a chain model for $\Omega M$. Adams' result remains valid if we use the complete DG coalgebra $C(M)$. Also the identity map $\tau: C \rightarrow \hat{\Omega}(C): \alpha \mapsto$ $[\alpha]$ is a twisting cochain, which exactly models the one of Brown. Therefore, the 
twisted tensor product

$$
C \hat{\otimes} \hat{\Omega}(C)
$$

with the twisted differential, which is the differential $b$ given in equation (3.1), gives the chain complex model of $L M$.

Note that the Whitney polynomial forms $A(M)$ embed into $C(M)$; thus we may form another chain complex

$A \hat{\otimes} \hat{\Omega}(C)$

with differential $b$ given by

$$
\begin{aligned}
& b\left(x \otimes\left[a_{1}|\cdots| a_{n}\right]\right) \\
:= & d x \otimes\left[a_{1}|\cdots| a_{n}\right]-\sum_{i}(-1)^{|x|+\left|\left[a_{1}|\cdots| a_{i-1}\right]\right|} x \otimes\left[a_{1}|\cdots| d a_{i}|\cdots| a_{n}\right] \\
- & \sum_{i} \sum_{\left(a_{i}\right)}(-1)^{|x|+\left|\left[a_{1}|\cdots| a_{i-1} \mid a_{i}^{\prime}\right]\right|} x \otimes\left[a_{1}|\cdots| a_{i}^{\prime}\left|a_{i}^{\prime \prime}\right| \cdots \mid a_{n}\right] \\
+ & \sum_{i}(-1)^{|x|+\left|y_{i}\right|} x y_{i} \otimes\left(\left[y_{i}^{*}\left|a_{1}\right| \cdots \mid a_{n}\right]-(-1)^{\left(\left|y_{i}\right|-1\right)\left|\left[a_{1}|\cdots| a_{n}\right]\right|}\left[a_{1}|\cdots| a_{n} \mid y_{i}^{*}\right]\right) .
\end{aligned}
$$

One can easily check that $b^{2}=0$. Comparing with equation (2.5), we see that it is also a twisted tensor product, and by the comparison theorem of spectral sequences for twisted tensor products (see e.g. McCleary [28], p. 224), we have that

$$
\iota \hat{\otimes} i d: A \hat{\otimes} \hat{\Omega}(C) \longrightarrow C \hat{\otimes} \hat{\Omega}(C)
$$

is in fact a quasi-isomorphism.

\section{The Chas-Sullivan loop product}

In this section we give a model of the Chas-Sullivan loop product defined in string topology.

Lemma 4.1. Let $(A, C, \iota)$ be a DG open Frobenius-like algebra. Define a product

$$
\text { • : } A \hat{\otimes} \hat{\Omega}(C) \otimes A \hat{\otimes} \hat{\Omega}(C) \longrightarrow A \hat{\otimes} \hat{\Omega}(C)
$$

by

$\left(x \otimes\left[a_{1}|\cdots| a_{n}\right]\right) \bullet\left(y \otimes\left[b_{1}|\cdots| b_{m}\right]\right):=(-1)^{|y|\left|\left[a_{1}|\cdots| a_{n}\right]\right|} x \wedge y \otimes\left[a_{1}|\cdots| a_{n}\left|b_{1}\right| \cdots \mid b_{m}\right]$.

Then $(A \hat{\otimes} \hat{\Omega}(C), \bullet, b)$ forms a DG algebra.

Proof. From the definition we see that $\bullet$ is associative, so we only need to show that $b$ is a derivation. Denoting $x \otimes \alpha:=x \otimes\left[a_{1}|\cdots| a_{n}\right]$ and $y \otimes \beta:=y \otimes\left[b_{1}|\cdots| b_{m}\right]$ for short, up to sign, we have

$$
\begin{aligned}
& b((x \otimes \alpha) \bullet(y \otimes \beta)) \\
= & b(x y \otimes \alpha \cdot \beta) \\
= & d(x y) \otimes \alpha \cdot \beta+x y \otimes d(\alpha \cdot \beta) \\
+ & \sum(x y)^{\prime} \otimes \tau(x y)^{\prime \prime} \circ(\alpha \cdot \beta),
\end{aligned}
$$

where $\tau$ is the twisting cochain, which acts on $\hat{\Omega}(C)$ by the adjoint action, while

$$
\begin{aligned}
& b(x \otimes \alpha) \bullet(y \otimes \beta)+(x \otimes \alpha) \bullet b(y \otimes \beta) \\
=\quad & (d x) y \otimes \alpha \cdot \beta+x y \otimes d(\alpha) \cdot \beta
\end{aligned}
$$




$$
\begin{aligned}
& +\sum x^{\prime} y \otimes\left(\tau x^{\prime \prime} \circ \alpha\right) \cdot \beta \\
& +\quad x(d y) \otimes \alpha \cdot \beta+x y \otimes \alpha \cdot d(\beta) \\
& +\sum x \cdot y^{\prime} \otimes \alpha \cdot\left(\tau y^{\prime \prime} \circ \beta\right) .
\end{aligned}
$$

To show that $(4.2)=(4.5)$, noting that $(4.3)=(4.6)+(4.8)$, we only need to show that (4.4) $=(4.7)+(4.9)$, i.e.

$$
\sum(x y)^{\prime} \otimes \tau(x y)^{\prime \prime} \circ(\alpha \cdot \beta)=\sum x^{\prime} y \otimes\left(\tau x^{\prime \prime} \circ \alpha\right) \cdot \beta+\sum x y^{\prime} \otimes \alpha \cdot\left(\tau y^{\prime \prime} \circ \beta\right) .
$$

By the Frobenius-like equation (2.4) it is equivalent for us to show

$$
\tau z \circ(\alpha \cdot \beta)=(\tau z \circ \alpha) \cdot \beta+\alpha \cdot(\tau z \circ \beta)
$$

where $z=(x y)^{\prime \prime}$. However, since all the $\tau z$ 's are primitive (for the definition and properties of primitive elements of a complete Hopf algebra, see e.g., Quillen [33], Appendix A.2) and a direct calculation shows that the primitive elements act as derivations, the result follows.

Now let us briefly recall the loop product defined in [6]. For the free loop space $L M$ of a manifold $M$, denote by $C_{*}(L M)$ the chain complex of the total space. For a $v \in C_{*}(L M)$, suppose it is not a linear combination of some other chains. Then we may associate to $v$ a chain $x \in C_{*}(M)$, which is the chain of marked points of $v$, and is called the "shadow" of $v$. Extend the map $v \mapsto x$ linearly to all elements in $C_{*}(L M)$. From the twisted tensor product point of view, the shadow can be understood as follows: suppose $x \otimes \alpha \in C_{*}(M) \otimes C_{*}(\Omega M)$ (with the twisted differential) represents $v \in C_{*}(L M)$; then $x \in C_{*}(M)$ is the shadow of $v$. Note that in general $v \mapsto x$ is not a chain map (as it does not preserve the dimension).

Now, for $u, v \in C_{*}(L M)$ two chains in general position, the loop product of $u$ and $v$ is defined as follows: first intersect their shadows $x$ and $y$ in $M$, then over the intersection set, do the Pontrjagin product pointwisely. From this we get a chain in $C_{*}(L M)$, denoted by $u \bullet v$, which is usually called the loop product of $u$ and $v$ :

$$
\begin{array}{cl}
\bullet: C_{*}(L M) \otimes C_{*}(L M) & \longrightarrow C_{*}(L M), \\
u \otimes v & \longmapsto u \bullet .
\end{array}
$$

Chas-Sullivan showed that $\partial$ is a derivation with respect to $\bullet$. Note that the loop product is partially defined only for two chains in general position. A theorem of Wilson [43] says that although the above product is defined on the chains in general position, it already captures all the homology information of $C_{*}(L M)$, and thus the loop product is well defined on the homology space $H_{*}(L M)$. Denote $\mathbb{H}_{*}(L M):=H_{*}(L M)[n]$ (which is called the loop homology of $\left.M\right)$; then $\mathbb{H}_{*}(L M)$ is a graded algebra with the product having degree 0 .

Theorem 4.2 (Identification of the loop product). Let $M$ be a simply connected, smooth closed manifold. Then the product $\bullet$ in Lemma 4.1 is quasi-isomorphic to the loop product in [6].

Proof. Denote by

$$
\psi: A \hat{\otimes} \hat{\Omega}(C) \longrightarrow C_{*}(L M)[n]
$$

the quasi-isomorphism of chain complexes via the zigzag

$$
A \hat{\otimes} \hat{\Omega}(C) \stackrel{\iota \hat{\otimes} i d}{\longrightarrow} C \hat{\otimes} \hat{\Omega}(C)=\widehat{C C}_{*}(C) \stackrel{\phi}{\longleftarrow} C(L M([m])
$$

(cf. Theorem 3.1 and equation (3.8)). We show that $\psi$ preserves the products. 
First let us consider $\psi(x \otimes \alpha)$ and $\psi(y \otimes \beta)$. From the twisted tensor product point of view, $x \otimes \alpha$ and $y \otimes \beta$ are two chains in $L M$ whose shadows are exactly $x$ and $y$ (see the above section), where $x$ and $y$ are now viewed as currents via the embedding $A \rightarrow C$. In general the intersection of two currents is not defined; however, if they come from two Whitney forms, say $x$ and $y$, then the intersection of $x$ and $y$ is exactly $x \wedge y$ (recall that on the homology level, the intersection of two homology classes is the Poincaré dual of the cup product of their Poincaré duals, and in our setting, the Poincaré dual is exactly induced from the embedding of the Whitney forms into the currents).

By the definition of the loop product, $\psi(x \otimes \alpha) \bullet \psi(y \otimes \beta)$ is a chain in $L M$ which is described as follows: The shadow is $x \wedge y=x y$ (the intersection), and for any point $q$ in the intersection, suppose there is a path $\gamma$ connecting $p$ and $q$ ( $p$ is the base point; see equation 3.7), i.e.

$$
\gamma:[0,1] \longrightarrow x y \subset M, \quad \gamma(0)=q, \quad \gamma(1)=p .
$$

Then by naturality of the twisting cochain, the fiber over $q$ is the Pontrjagin product

$$
\gamma_{\#}(\alpha) \cdot \gamma_{\#}(\beta),
$$

where $\gamma_{\#}$ is the chain map induced from

$$
\begin{aligned}
\gamma: \Omega_{p} M & \longrightarrow \Omega_{q} M, \\
\alpha & \longmapsto \gamma \cdot \alpha \cdot \gamma^{-1} .
\end{aligned}
$$

On the other hand, $\psi\left((-1)^{|\alpha||y|} x y \otimes \alpha \cdot \beta\right)$ is a chain in $L M$ described as follows: its shadow is also $x y$, and the fiber over $q$ is

$$
\gamma_{\#}(\alpha \cdot \beta) \text {. }
$$

In order to show that

$$
\psi(x \otimes \alpha) \bullet \psi(y \otimes \beta)=\psi\left((-1)^{|\alpha||y|} x y \otimes \alpha \cdot \beta\right),
$$

we only need to show that $(4.10)=4.12$ :

$$
\gamma_{\#}(\alpha) \cdot \gamma_{\#}(\beta)=\gamma_{\#}(\alpha \cdot \beta) .
$$

However, looking at the path action (4.11), we have

$$
\gamma(\alpha \cdot \beta)=\gamma(\alpha) \cdot \gamma(\beta),
$$

for any $\alpha, \beta \in \Omega_{p} M$, and on the chain level, it exactly gives equality (4.13).

\section{Commutativity of the loop product AND THE GERSTENHABER ALGEBRA}

In [6, Chas and Sullivan showed that at the chain level, the loop product is not commutative but homotopy commutative, and hence the loop homology is commutative. Such a homotopy operator gives a pre-Lie algebra on the loop homology, which was originally defined on the Hochschild cochain complex of an associative algebra (see Gerstenhaber [16]). The loop homology with the loop product and the commutator of the pre-Lie operator forms a Gerstenhaber algebra.

We first give a description of the pre-Lie operator $*$ defined in [6]: for two chains $\alpha, \beta \in C_{*}(L M)$ in general position, we have that $\tilde{\alpha}$ is a transversal to loops in $\beta$. Form a chain $\alpha * \beta$ given by the following loops: for any loop $\gamma$ in $\beta$, first go around $\gamma$ from the base point till the intersection point with $\tilde{\alpha}$, then go around the loops 
in $\alpha$, and finally go around the rest of $\gamma$. Such a star $*$-operator can be modeled as follows:

Lemma 5.1. Let $(A, C, \iota)$ be as before. Define an operator

$$
\text { * : } A \hat{\otimes} \hat{\Omega}(C) \otimes A \hat{\otimes} \hat{\Omega}(C) \longrightarrow A \hat{\otimes} \hat{\Omega}(C)
$$

as follows: for $\alpha=x \otimes\left[a_{1}|\cdots| a_{n}\right], \beta=y \otimes\left[b_{1}|\cdots| b_{m}\right] \in A \hat{\otimes} \hat{\Omega}(C)$,

$\alpha * \beta=\sum_{i=1}^{n}(-1)^{|y|+(|\beta|-1)\left|\left[a_{i+1}|\cdots| a_{n}\right]\right|} \varepsilon\left(a_{i} y\right) x \otimes\left[a_{1}|\cdots| a_{i-1}\left|b_{1}\right| \cdots\left|b_{m}\right| a_{i+1}|\cdots| a_{n}\right]$, where $\varepsilon$ is the counit of $C$. Then,

$$
b(\alpha * \beta)=b \alpha * \beta+(-1)^{|\alpha|+1} \alpha * b \beta+(-1)^{|\alpha|}\left(\alpha \bullet \beta-(-1)^{|\alpha||\beta|} \beta \bullet \alpha\right) .
$$

In particular, $\left(H_{*}(A \hat{\otimes} \hat{\Omega}(C)), \bullet\right)$ is a graded commutative algebra.

Proof. The proof is more or less the same as Gerstenhaber [16], Theorem 3; hence we omit it.

Definition 5.2 (Pre-Lie algebra). Let $V$ be a graded vector space over $k$. A pre-Lie structure on $V$ is a degree-one binary operator

$$
*: V \otimes V \longrightarrow V
$$

such that

(5.3) $(\gamma * \alpha) * \beta-(-1)^{(|\alpha|+1)(|\beta|+1)}(\gamma * \beta) * \alpha=\gamma *\left(\alpha * \beta-(-1)^{(|\alpha|+1)(|\beta|+1)} \beta * \alpha\right)$.

We call $(V, *)$ a pre-Lie algebra, or a pre-Lie system.

Lemma 5.3. Let $(V, *)$ be a pre-Lie algebra. Define

$$
\begin{aligned}
\{,\}: V \otimes V & \longrightarrow V \\
a \otimes b & \longmapsto a * b-(-1)^{(|a|+1)(|b|+1)} b * a .
\end{aligned}
$$

Then $(V,\{\}$,$) is a degree-one Lie algebra.$

Proof. See Gerstenhaber [16], Theorem 1.

Lemma 5.4. Let $(A, C, \iota)$ be as above. Then $(A \hat{\otimes} \hat{\Omega}(C), *)$ is a pre-Lie algebra.

Proof. We also omit the proof; one may refer to Gerstenhaber [16], Sections 5-7.

Corollary 5.5. Let $(A, C, \iota)$ be as above. Then

$$
(A \hat{\otimes} \hat{\Omega}(C),\{,\}, b)
$$

is a degree-one $D G$ Lie algebra. In particular, $\left(H_{*}(A \hat{\otimes} \hat{\Omega}(C)),\{\},\right)$ is a degree-one graded Lie algebra.

Proof. The degree-one Lie algebra follows from the above lemma and the theorem of Gerstenhaber (Lemma 5.3). Lemma 5.1 shows that $b$ respects $\{$,$\} .$

Definition 5.6 (Gerstenhaber algebra). Let $V$ be a graded vector space over a field $k$. A Gerstenhaber algebra on $V$ is a triple $(V, \cdot,\{\}$,$) such that$

(1) $(V, \cdot)$ is a graded commutative algebra;

(2) $(V,\{\}$,$) is a graded degree-one Lie algebra;$

(3) the bracket is a derivation for both variables. 
We are now ready to model the theorem of $[\underline{6}$, where the Lie bracket $\{$,$\} is called$ the loop bracket:

Theorem 5.7 (Gerstenhaber algebra of the free loop space). Let $M$ be a simply connected, smooth closed manifold and LM its free loop space. Then

$$
\left(H_{*}(A \hat{\otimes} \hat{\Omega}(C)), \bullet,\{,\}\right)
$$

is a Gerstenhaber algebra and is isomorphic to the Gerstenhaber algebra on $\mathbb{H}_{*}(L M)$ obtained in [6].

Proof. We have shown that $H_{*}(A \hat{\otimes} \hat{\Omega}(C))$ is a graded commutative algebra (Lemma 5.1) and a degree-one graded Lie algebra (Corollary 5.5). Next we show that the bracket is a derivation with respect to the loop product for both variables. By symmetry we only need to show, for $\alpha, \beta, \gamma \in H_{*}(A \hat{\otimes} \hat{\Omega}(C))$,

$$
\{\alpha \bullet \beta, \gamma\}=\alpha \bullet\{\beta, \gamma\}+(-1)^{|\beta|(|\gamma|+1)}\{\alpha, \gamma\} \bullet \beta .
$$

This immediately follows from the following Lemma 5.8

As we shall see later (Section77), both $\mathbb{H}_{*}(L M)$ and $H_{*}(A \hat{\otimes} \Omega(C)$ ) have a BatalinVilkovisky algebra structure, and $\{$,$\} is completely determined by the Batalin-$ Vilkovisky differential operator. The identification of the Batalin-Vilkovisky algebras (Theorem 17.4) then gives the identification of the Gerstenhaber algebras of $\mathbb{H}_{*}(L M)$ and $H_{*}(A \hat{\otimes} \hat{\Omega}(C))$. However, the identification of $\{$,$\} cannot be obtained$ directly from the above arguments, even though we followed Gerstenhaber [16] and Chas-Sullivan [6] step by step, since it comes from the commutator of the homotopy operator $*$, which is a priori not a chain map.

Lemma 5.8. Let $A$ be as above. Then for $\alpha=x \otimes\left[a_{1}|\cdots| a_{n}\right], \beta=y \otimes\left[b_{1}|\cdots| b_{m}\right]$, $\gamma=z \otimes\left[c_{1}|\cdots| c_{l}\right] \in A \hat{\otimes} \hat{\Omega}(C)$,

(1) $(\alpha \bullet \beta) * \gamma=\alpha \bullet(\beta * \gamma)+(-1)^{|\beta|(|\gamma|+1)}(\alpha * \gamma) \bullet \beta$;

(2) setting

$$
\begin{gathered}
h(\gamma \otimes \alpha \otimes \beta)=\sum_{i<j}(-1)^{\epsilon} \varepsilon\left(c_{i} x\right) \varepsilon\left(c_{j} y\right) z \\
\otimes\left[c_{1}|\cdots| c_{i-1}\left|a_{1}\right| \cdots\left|a_{n}\right| c_{i+1}|\cdots| c_{j-1}\left|b_{1}\right| \cdots\left|b_{m}\right| c_{j+1}|\cdots| c_{l}\right], \\
\text { where } \epsilon=(|\alpha|-1)\left|\left[c_{i+1}|\cdots| c_{l}\right]\right|+(|\beta|-1)\left|\left[c_{j+1}|\cdots| c_{l}\right]\right|, \text { we have } \\
(b \circ h-h \circ b)(\gamma \otimes \alpha \otimes \beta)=\gamma *(\alpha \bullet \beta)-(\gamma * \alpha) \bullet \beta-(-1)^{|\alpha|(|\gamma|+1)} \alpha \bullet(\gamma * \beta) .
\end{gathered}
$$

The above lemma is similar to [6], Lemma 4.6, with a minor modification, whose proof is deferred to the Appendix.

\section{IsOMORPHISM OF TWO GERSTENHABER ALGEBRAS}

The notion of a Gerstenhaber algebra was first introduced by Gerstenhaber in his study of the deformation theory of associative algebras. In [16, Gerstenhaber showed that the Hochschild cohomology of an associative algebra is endowed with the structure of a Gerstenhaber algebra. As a direct application, the Hochschild cohomology of the cochain algebra $C^{*}(M)$ of a manifold is a Gerstenhaber algebra. As we have seen, the (co)homology of the free loop space is closely related to the appropriate Hochschild homology of the (co)chain algebra; one wonders if the Gerstenhaber algebra deduced from Gerstenhaber's result is identical to the one discovered in string topology. 
Such a problem has been discussed and proved by Cohen-Jones [10, Tradler [37, Merkulov [32, Félix-Thomas-Vigué-Poirrier [15] and McClure [29. Here we also offer an affirmative answer via our chain model of the free loop space. Recall the results of Gerstenhaber in [16]:

Definition 6.1 (Product and bracket of the Hochschild cochain complex). Let $A$ be a (DG) algebra over a field $k$ and let

$$
H C^{*}(A ; A)=\operatorname{Hom}\left(\bigoplus_{n=0}^{\infty} A^{\otimes n}, A\right)
$$

be its Hochschild cochain complex. Define the product $U$, the pre-Lie operator $*$, and the bracket $\{$,$\} on H C^{*}(A ; A)$ as follows: for $f \in \operatorname{Hom}\left(A^{\otimes n} ; A\right)$, $g \in \operatorname{Hom}\left(A^{\otimes m} ; A\right)$, up to sign,

(1) $f \cup g \in \operatorname{Hom}\left(A^{\otimes m+n}, A\right)$ : for any $a_{1}, \cdots, a_{m+n} \in A$,

$$
(f \cup g)\left(a_{1}, \cdots, a_{m+n}\right):=f\left(a_{1}, \cdots, a_{n}\right) \cdot g\left(a_{n+1}, \cdots, a_{m+n}\right) ;
$$

(2) $f * g \in \operatorname{Hom}\left(A^{\otimes m+n-1}, A\right)$ : for any $a_{1}, \cdots, a_{n+m-1} \in A$,

$$
(f * g)\left(a_{1}, \cdots, a_{n+m-1}\right):=\sum_{i=1}^{n} \pm f\left(a_{1}, \cdots, a_{i-1}, g\left(a_{i}, \cdots, a_{i+m-1}\right), \cdots, a_{n+m-1}\right) ;
$$

(3) $\{f, g\} \in \operatorname{Hom}\left(A^{\otimes m+n-1}, A\right)$ is the commutator of $*$ :

$$
\{f, g\}:=f * g-(-1)^{(|f|+1)(|g|+1)} g * f .
$$

Gerstenhaber's main statement in [16] is the following theorem:

Theorem 6.2 (Gerstenhaber). Let $A$ be a $D G$ associative algebra over a field $k$ and let the operators $\cup, *$ and $\{$,$\} be given in the above definition; then Lemmas$ 5.1 and 5.8 hold. Therefore the Hochschild cohomology $\left(H H^{*}(A ; A), \cup,\{\},\right)$ is a Gerstenhaber algebra.

The following theorem is inspired by the results of the authors mentioned at the beginning of this section:

Theorem 6.3 (Isomorphism of two Gerstenhaber algebras). Let $M$ be a simply connected manifold and let $A$ be the Whitney forms on $M$. Then

$$
\mathbb{H}_{*}(L M) \stackrel{\cong}{\longrightarrow} H H^{*}(A ; A)
$$

are isomorphic as Gerstenhaber algebras.

Proof. In fact, let $C$ be the set of currents on $M$; then the Hochschild cochain complex is chain equivalent to $A \hat{\otimes} \hat{\Omega}(C)$ :

$$
H C^{*}(A ; A) \simeq A \hat{\otimes} \hat{\Omega}(C) .
$$

For homogeneous $f, g \in H C^{*}(A ; A)$, we may write them as $f=x \otimes\left[a_{1}|\cdots| a_{n}\right], g=$ $y \otimes\left[b_{1}|\cdots| b_{m}\right] \in A \hat{\otimes} \hat{\Omega}(C)$; the operators $\cdot, *$ and $\{$,$\} defined above by (6.1), (6.2)$ and (6.3) can be rewritten as

$$
f \cup g=x \cdot y \otimes\left[a_{1}|\cdots| a_{n}\left|b_{1}\right| \cdots \mid b_{m}\right]
$$

and

$$
f * g=\sum_{i=1}^{n}\left\langle a_{i}, y\right\rangle x \otimes\left[a_{1}|\cdots| a_{i-1}\left|b_{1}\right| \cdots\left|b_{m}\right| a_{i+1}|\cdots| a_{n}\right]
$$


and

$$
\{f, g\}:=f * g-(-1)^{(|f|+1)(|g|+1)} g * f .
$$

Comparing them with the loop product (4.1) and pre-Lie operator (5.1), we see that $\mathbb{H}_{*}(L M)$ and $H H^{*}(A ; A)$ are isomorphic as Gerstenhaber algebras.

Remark 6.4. In the paper of Voronov and Gerstenhaber [40, the authors show that the Hochschild cochain complex has a very ample structure, which they called the homotopy Gerstenhaber algebra, where a family of new operators besides the pre-Lie operator is introduced: while the pre-Lie operator gives the homotopy of the commutativity, these new operators give all the higher homotopies. From the above proof we may see that the chain complex of the free loop space of a manifold also bears the structure of a homotopy Gerstenhaber algebra, which is highly related to the cactus operad and the little disk operad (see Voronov [38] and [39]).

\section{7. $S^{1}$-ACTiOn AND the Batalin-Vilkovisky ALGEBRA}

Let $J$ be the $S^{1}$-action on the loop homology. In [6], Chas and Sullivan prove that $\left(\mathbb{H}_{*}(L M), \bullet, J\right)$ forms a Batalin-Vilkovisky algebra. Namely, $J$ on homology is not a derivation with respect to $\bullet$, but the deviation from being a derivation is a derivation. One deduces that

$$
\{a, b\}:=(-1)^{|\alpha|} J(\alpha \bullet \beta)-(-1)^{|\alpha|} J(\alpha) \bullet b-\alpha \bullet J(\beta), \quad \text { for all } \quad \alpha, \beta \in \mathbb{H}_{*}(L M),
$$

defines a degree-one graded Lie algebra on $\mathbb{H}_{*}(L M)$, which is in fact the loop bracket on homology.

Definition 7.1 (Batalin-Vilkovisky algebra). Let $V$ be a graded vector space over a field $k$. A Batalin-Vilkovisky algebra on $V$ is a triple $(V, \bullet, \Delta)$ such that:

(1) $(V, \bullet)$ is a graded commutative algebra.

(2) $\Delta: V \rightarrow V$ is a degree-one operator with $\Delta^{2}=0$.

(3) The deviation from being a derivation of $\Delta$ with respect to $\bullet$ is a derivation for both variables; namely,

$$
(-1)^{|\alpha|} \Delta(\alpha \bullet \beta)-(-1)^{|\alpha|} \Delta(\alpha) \bullet b-\alpha \bullet \Delta(\beta)
$$

is a derivation for both $\alpha, \beta \in V$.

A Batalin-Vilkovisky algebra is a special kind of Gerstenhaber algebra.

Proposition 7.2. Let $(V, \bullet, \Delta)$ be a Batalin-Vilkovisky algebra. Define $[]:, V \otimes$ $V \longrightarrow V$ by

$$
[\alpha, \beta]:=(-1)^{|\alpha|} \Delta(\alpha \bullet \beta)-(-1)^{|\alpha|} \Delta(\alpha) \bullet b-\alpha \bullet \Delta(\beta), \text { for } \alpha, \beta \in V .
$$

Then $(V, \bullet,[]$,$) forms a Gerstenhaber algebra.$

Proof. See Getzler [17, Proposition 1.2.

Lemma 7.3. Let $M$ be a simply connected manifold and $L M$ be its free loop space. Then

(7.1) $\{\alpha, \beta\}=(-1)^{|\alpha|} J(\alpha \bullet \beta)-(-1)^{|\alpha|} J(\alpha) \bullet b-\alpha \bullet J(\beta)$, for $\alpha, \beta \in \mathbb{H}_{*}(L M)$, where $\{$,$\} and \bullet$ are the loop bracket and the loop product, respectively, and $J$ is the induced $S^{1}$-action on $\mathbb{H}_{*}(L M)$. 
More precisely, let $(A, C, \iota)$ be the $D G$ open Frobenius-like algebra of $M$ and $A \hat{\otimes} \hat{\Omega}(C)$ be the twisted tensor product, and let $B$ be the dual of Connes' cyclic operator on $C \hat{\otimes} \hat{\Omega}(C)$. Then there is a linear map

$$
h: A \hat{\otimes} \hat{\Omega}(C) \otimes A \hat{\otimes} \hat{\Omega}(C) \longrightarrow C \hat{\otimes} \hat{\Omega}(C)
$$

such that for any $\alpha, \beta \in A \hat{\otimes} \hat{\Omega}(C)$,

$$
(b \circ h-h \circ b)(\alpha \otimes \beta)=\{\alpha, \beta\}-(-1)^{|\alpha|} B(\alpha \bullet \beta)-(-1)^{(|\beta|+1)(|\alpha|+1)} \beta \bullet B(\alpha)+\alpha \bullet B(\beta) .
$$

The above lemma is similar to Lemma 5.2 in [6], whose proof is also given in the Appendix. By this lemma we obtain:

Theorem 7.4 (Batalin-Vilkovisky algebra of the free loop space). Let $M$ be a simply connected, smooth closed manifold and let $A$ be the Whitney forms and $C$ be the currents on $M$. Then

$$
\left(H_{*}(A \hat{\otimes} \hat{\Omega}(C)), \bullet, B\right)
$$

is a Batalin-Vilkovisky algebra, and is isomorphic to the Batalin-Vilkovisky algebra on $\mathbb{H}_{*}(L M)$ obtained in [6].

Proof. We have shown (Theorem 5.7) that

$$
\left(H_{*}(A \hat{\otimes} \hat{\Omega}(C)), \bullet,\{,\}\right)
$$

is a Gerstenhaber algebra, and therefore the loop bracket $\{$,$\} is a derivation for$ both variables with respect to $\bullet$. Lemma 7.3 says that the deviation of $B$ from being a derivation is exactly the loop bracket. Thus, according to Definition 7.1 ,

$$
\left(H_{*}(A \hat{\otimes} \hat{\Omega}(C)), \bullet, B\right)
$$

is a Batalin-Vilkovisky algebra.

In Theorem 3.2 we identified $H_{*}(L M)$ with $H_{*}\left(\widehat{C C}_{*}(C), b\right)$ and hence with $H_{*}(A \hat{\otimes} \hat{\Omega}(C), b)$ (up to a degree shifting) as vector spaces, together with the identification of the $S^{1}$-rotation with Connes' cyclic operator $B$. In Theorem 4.2 we identified the loop product with the product on $H_{*}(A \hat{\otimes} \hat{\Omega}(C), b)$. From the definition, a Batalin-Vilkovisky algebra is completely determined by these two operations, and therefore the Batalin-Vilkovisky algebra obtained above models the one of string topology.

\section{EquiVARIANT HOMOLOGY AND THE GRAVITY ALGEBRA}

In Chas-Sullivan [6], the authors also showed that the equivariant homology of the free loop space, $H_{*}^{S^{1}}(L M)$, forms a Lie algebra. Later in [7] they continued to show that the equivariant homology is endowed with more structures, for example, the gravity algebra. Recall that the equivariant homology $H_{*}^{S^{1}}(L M)$ of $L M$ is defined to be the homology of $E S^{1} \times_{S^{1}} L M$, where $E S^{1}$ is the universal bundle over the classifying space $B S^{1}$. There is a fibration

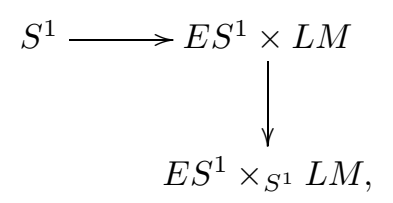


and the associated Gysin sequence is given by

$$
\begin{aligned}
\cdots \longrightarrow H_{*}\left(E S^{1} \times L M\right) \longrightarrow H_{*}^{S^{1}}(L M) \longrightarrow & H_{*-2}^{S^{1}}(L M) \\
& \longrightarrow H_{*-1}\left(E S^{1} \times L M\right) \longrightarrow \cdots .
\end{aligned}
$$

By identifying $H_{*}\left(E S^{1} \times L M\right)$ with $H_{*}(L M)$, we obtain

$$
\cdots \longrightarrow H_{*}(L M) \stackrel{E}{\longrightarrow} H_{*}^{S^{1}}(L M) \longrightarrow H_{*-2}^{S^{1}}(L M) \stackrel{M}{\longrightarrow} H_{*-1}(L M) \longrightarrow \cdots,
$$

where $E$ and $M$ can be interpreted as "forgetting" and "adding" the marked points on the loops of the corresponding spaces.

Topologically $M \circ E$ is exactly the $S^{1}$-operation $J$ on homology $H_{*}(L M)$, and $E \circ M=0$. Now for $a_{1}, a_{2} \in H_{*}^{S^{1}}(L M)$, define

$$
\left\{a_{1}, a_{2}\right\}:=(-1)^{\left|a_{1}\right|+2-n} E\left(M\left(a_{1}\right) \bullet M\left(a_{2}\right)\right),
$$

which is usually called the string bracket. Then $\{$,$\} thus defined in fact gives$ on $H_{*}^{S^{1}}(L M)$ a degree $2-n$ graded Lie algebra structure. Moreover, $H_{*}^{S^{1}}(L M)$ satisfies the generalized Jacobi identity and hence forms a gravity algebra in the sense of Getzler [18]:

Definition 8.1 (Gravity algebra). Let $V$ be a chain complex over a field $k$. A gravity algebra on $V$ is a sequence of graded skew-symmetric operators:

$$
c_{n}: V^{\otimes n} \longrightarrow V, \quad \text { for } n \geq 2,
$$

of degree $2-n$, satisfying the following relations: if $k>2$ and $l \geq 0$, and $a_{1}, \cdots, a_{k}, b_{1}, \cdots, b_{l} \in V$, then

$$
\begin{aligned}
& \sum_{1 \leq i<j \leq k}(-1)^{\epsilon}\left\{\left\{a_{i}, a_{j}\right\}, a_{1}, \cdots, \widehat{a_{i}}, \cdots, \widehat{a_{j}}, \cdots, a_{k}, b_{1}, \cdots, b_{l}\right\} \\
= & \begin{cases}\left\{\left\{a_{1}, \cdots, a_{k}\right\}, b_{1}, \cdots, b_{l}\right\}, & l>0, \\
0, & l=0,\end{cases}
\end{aligned}
$$

where we write $c_{n}\left(a_{1}, \cdots, a_{n}\right)$ as $\left\{a_{1}, \cdots, a_{n}\right\}$, and $\epsilon=\left|a_{i}\right|\left(\left|a_{i}\right|+\cdots+\left|a_{i-1}\right|\right)+$ $\left|a_{j}\right|\left(\left|a_{1}\right|+\cdots+\left|\widehat{a_{i}}\right|+\cdots+\left|a_{j-1}\right|\right)$.

A gravity algebra contains a Lie algebra: let $k=3$ and $l=0$; then equation (8.1) is exactly the Jacobi identity. For more details of the gravity algebra on the equivariant homology $H_{*}^{S^{1}}(L M)$, see [6], [7], 35] or Theorem 8.5 below. The above construction is rather topological, but we can see this algebraically from the cyclic homology of A. Connes.

Definition 8.2 (Cyclic homology of a coalgebra). Let $C$ be a DG coalgebra. The cyclic homology of $C$, denoted by $C H_{*}(C)$, is the homology of the chain complex $C C_{*}(C)\left[u, u^{-1}\right] /\left\langle u^{-1}\right\rangle$, where $u$ is a parameter of degree 2 , with differential induced from the one defined on $C C_{*}(C)\left[u, u^{-1}\right]$ :

$$
\begin{aligned}
b+u^{-1} B: \quad C C_{*}(C)\left[u, u^{-1}\right] & \longrightarrow C C_{*}(C)\left[u, u^{-1}\right], \\
x \otimes u^{n} & \longmapsto b x \otimes u^{n}+B x \otimes u^{n-1} .
\end{aligned}
$$


According to Jones 22], there are several cyclic homology theories. Here we adopt the most common one used in the literature. The above definition can be generalized to the complete DG coalgebra case.

Theorem 8.3 (Connes' exact sequence and the Gysin sequence). (1) Let $C$ be a $D G$ cocommutative coalgebra. Then there is a long exact sequence, called Connes' exact sequence:

$$
\cdots \longrightarrow H_{*}(C) \stackrel{E}{\longrightarrow} C H_{*}(C) \longrightarrow C H_{*-2}(C) \stackrel{M}{\longrightarrow} H H_{*-1}(C) \longrightarrow \cdots
$$

(2) If, moreover, $C$ is the DG coalgebra of a simply connected manifold $M$, then the following diagram is commutative:

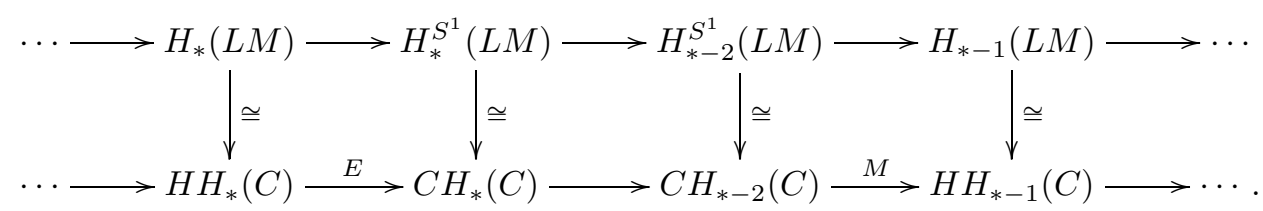

Proof. The proof of the two statements is the coalgebra analogue of Loday 25. Theorem 7.2.3, p. 235]. In fact, observe that we have a short exact sequence:

$$
0 \longrightarrow C C_{*}(C) \longrightarrow C C_{*}(C)\left[u, u^{-1}\right] /\left\langle u^{-1}\right\rangle \stackrel{\cdot u^{-1}}{\longrightarrow} C C_{*}(C)\left[u, u^{-1}\right] /\left\langle u^{-1}\right\rangle \longrightarrow 0 .
$$

The associated long exact sequence on homology is exactly Connes' long exact sequence. The isomorphism between

$$
H_{*}^{S^{1}}(L M) \stackrel{\cong}{\longrightarrow} C H_{*}(C)
$$

comes from the fact that $C_{*}^{S^{1}}(L M)$ is chain equivalent to (see Jones [22])

$$
\left(C_{*}(L M)\left[u, u^{-1}\right] /\left\langle u^{-1}\right\rangle, b+u^{-1} J\right) .
$$

Applying Theorem 3.2 gives the desired isomorphism.

Lemma 8.4. In the long exact sequence (8.2) of the above theorem,

$$
M \circ E=B: H H_{*}(C) \longrightarrow H H_{*+1}(C) .
$$

Proof. The statement follows from chasing the diagram of the short exact sequence

$$
0 \longrightarrow \widehat{C C}_{*}(C) \longrightarrow \widehat{C C}\left[u, u^{-1}\right] / u^{-1} \stackrel{\cdot u^{-1}}{\longrightarrow} \widehat{C C}_{*}(C)\left[u, u^{-1}\right] / u^{-1} \longrightarrow 0 .
$$

The check is left to the reader.

Theorem 8.5 (Gravity algebra on the free loop space). Let $M$ be a simply connected manifold and let $C$ be the DG coalgebra of $M$. Let $\mathbb{C H}_{*}(C):=C H_{*}(C)[n-2]$ and define

$$
\begin{aligned}
& c_{n}: \mathbb{C H}_{*}(C)^{\otimes n} \longrightarrow \mathbb{C H}_{*}(C), \\
& \alpha_{1} \otimes \cdots \otimes \alpha_{n} \longmapsto \\
&(-1)^{\epsilon} E\left(M\left(\alpha_{1}\right) \bullet \cdots \bullet M\left(\alpha_{n}\right)\right),
\end{aligned}
$$

where $\bullet$ is the loop product and $\epsilon=(n-1)\left|\alpha_{1}\right|+(n-2)\left|\alpha_{2}\right|+\cdots+\left|\alpha_{n-1}\right|$. Then $\left(\mathbb{C H}_{*}(C),\left\{c_{n}\right\}\right)$ is a gravity algebra. 
Proof. We have shown that $\left(H H_{*}(C), \bullet, B\right)$ is a Batalin-Vilkovisky algebra. $B$ being a second-order operator with respect to $\bullet$ implies that

$$
\begin{aligned}
B\left(x_{1} \bullet x_{2} \bullet \cdots \bullet x_{n}\right) & =\sum_{i<j} \pm B\left(x_{i} \bullet x_{j}\right) \bullet x_{1} \bullet \cdots \bullet \widehat{x_{i}} \bullet \cdots \bullet \widehat{x_{j}} \bullet \cdots \bullet x_{n} \\
& \pm(n-2) \sum_{i} x_{1} \bullet \cdots \bullet B x_{i} \bullet \cdots \bullet x_{n},
\end{aligned}
$$

for $x_{1}, \cdots, x_{n} \in H H_{*}(C)$.

Now let $x_{i}:=M\left(a_{i}\right)$ and apply $E$ on both sides of the above equality to obtain:

$$
\begin{aligned}
& E \circ B\left(M\left(a_{1}\right) \bullet M\left(a_{2}\right) \bullet \cdots \bullet M\left(a_{n}\right)\right) \\
& =\sum_{i<j} \pm E \circ\left(B\left(M\left(a_{i}\right) \bullet M\left(a_{j}\right)\right) \bullet M\left(a_{1}\right) \bullet \cdots \bullet \widehat{M\left(a_{i}\right)} \bullet \cdots \bullet \widehat{M\left(a_{j}\right)} \bullet \cdots \bullet M\left(a_{n}\right)\right) \\
& \pm(n-2) \sum_{i} E \circ\left(M\left(a_{1}\right) \bullet \cdots \bullet B \circ M\left(a_{i}\right) \bullet \cdots \bullet M\left(a_{n}\right)\right) .
\end{aligned}
$$

Note that $E \circ B=E \circ M \circ E=0$ and $B \circ M=M \circ E \circ M=0$ (above lemma), so we exactly have

$$
\sum_{1 \leq i<j \leq k} \pm\left\{\left\{a_{i}, a_{j}\right\}, a_{1}, \cdots, \widehat{a_{i}}, \cdots, \widehat{a_{j}}, \cdots, a_{k}\right\}=0 .
$$

Similarly by multiplying $y_{1} \bullet \cdots \bullet y_{l}$ to (8.3), letting $y_{j}:=M\left(b_{j}\right)$ and applying $E$ on both sides, we obtain

$$
\sum_{1 \leq i<j \leq k} \pm\left\{\left\{a_{i}, a_{j}\right\}, a_{1}, \cdots, \widehat{a_{i}}, \cdots, \widehat{a_{j}}, \cdots, a_{k}, b_{1}, \cdots, b_{l}\right\}=\left\{\left\{a_{1}, \cdots, a_{k}\right\}, b_{1}, \cdots, b_{l}\right\},
$$

for $l>0$. This proves the theorem.

\section{The NON-SIMPLY CONNECTED MANIFOLDS}

In the previous sections, we have only discussed the case when the manifold $M$ is simply connected. In this section we sketch the construction of string topology on a general non-simply connected manifold. The idea is to lift the loops on $M$ to their universal covering $\tilde{M}$, where the loops now becomes paths, which can be characterized explicitly. We learned this idea indirectly from Mike Mandell via a conversation with James McClure. The same idea has been espoused in, for example, Adem-Ruan [2] and Lupercio-Uribe-Xicotencatl [26].

We begin with the following observation about the free loop space $L M$.

Lemma 9.1 (Equivalent characterization of $L M$ ). Let $M$ be a smooth manifold. Denote by $G$ the fundamental group $\pi_{1}(M)$ and by $\tilde{M}$ the universal covering of $M$. For any $g \in G$, let

$$
L_{g} \tilde{M}:=\{f: I=[0,1] \rightarrow \tilde{M} \mid f(1)=g \circ f(0)\} .
$$

Then $\coprod_{g \in G} L_{g} \tilde{M}$ admits a G-action induced from that on $\tilde{M}$ : for $f \in L_{g} \tilde{M}$ and $h \in G$,

$$
\begin{aligned}
h \circ f:[0,1] & \longrightarrow \tilde{M}, \\
x & \longmapsto h \circ f(x) .
\end{aligned}
$$


Since $(h \circ f)(1)=h \circ f(1)=h \circ(g \circ f(0))=h g h^{-1} \circ((h \circ f)(0)), h \circ f \in L_{h g h^{-1}} \tilde{M}$. There is a homeomorphism

$$
\coprod_{g \in G} L_{g} \tilde{M} / G \cong L M
$$

and the following diagram commutes:

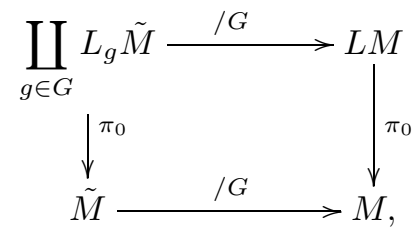

where $\pi_{0}$ is the projection of the paths to their starting points.

The proof of the lemma is a direct check. In the following we shall use this proposition to construct a chain complex model for $L M$ from $\coprod_{g} L_{g} \tilde{M}$. However, since $\tilde{M}$ may not be closed, the dual space of the Whitney polynomial differential forms may not compute the homology of $\tilde{M}$ correctly, and therefore may not be a correct chain model for $\tilde{M}$. However, if we denote by $A_{\sigma}^{p}$ the set of Whitney forms of degree less than or equal to $p$ on a cube $\sigma$ in $\tilde{M}$, then by the definition of the Whitney forms,

$$
A(\tilde{M})=\lim _{\leftarrow \sigma} \lim _{\sigma} A_{\sigma}^{p},
$$

where the inverse limit is taken over an increasing union of cubes in $M$. Let $C_{\sigma}^{p}:=\operatorname{Hom}\left(A_{\sigma}^{p}, \mathbb{Q}\right)$, and

$$
C(\tilde{M}):=\lim _{\sigma} \lim _{p} C_{\sigma}^{p} .
$$

$C(\tilde{M})$ may be viewed as the set of currents with compact support. Similar to the cochain case, there is a chain map

$$
\rho: C_{*}(\tilde{M}) \longrightarrow C(\tilde{M})
$$

from the singular chain complex $C_{*}(\tilde{M})$ to $C(\tilde{M})$ which is given by integration, inducing an isomorphism on the homology. Denote by $A_{\mathrm{c}}(\tilde{M})$ the set of Whitney forms with compact support. Then there is an embedding

$$
\begin{aligned}
\iota: A_{\mathrm{c}}(\tilde{M}) & \longrightarrow C(\tilde{M}), \\
\alpha & \longmapsto\left\{\beta \mapsto \int_{\tilde{M}} \alpha \wedge \beta\right\} .
\end{aligned}
$$

By the fact that $H_{\mathrm{c}}^{*}(\tilde{M}) \cong H_{*}(\tilde{M} ; \mathbb{Q})$ one deduces that

$$
\left(A_{\mathrm{c}}(\tilde{M}), C(\tilde{M}), \iota\right)
$$

is a DG open Frobenius-like algebra. Moreover, the action of $G$ on $\tilde{M}$ induces a $G$-action on $A_{\mathrm{c}}(\tilde{M})$ and $C(M)$, and the inclusion $\iota: A_{\mathrm{c}}(\tilde{M}) \longrightarrow C(\tilde{M})$ is in fact $G$-equivariant.

Recall the definition of $L_{g} \tilde{M}$ :

$$
L_{g} \tilde{M}=\{f:[0,1] \rightarrow \tilde{M} \mid f(1)=g \circ f(0)\} .
$$


Note that $L_{g} \tilde{M}$ is connected: given $f_{1}, f_{2} \in L_{g} \tilde{M}$, let $\gamma$ be a path in $\tilde{M}$ connecting $f_{1}(0)$ and $f_{2}(0)$. Then $g \circ \gamma$ is a path connecting $f_{1}(1)$ and $f_{2}(1)$, and $f_{1} \circ(g \circ \gamma) \circ$ $f_{2}^{-1} \circ \gamma^{-1}$ is a closed path in $\tilde{M}$. Since $\tilde{M}$ is simply connected, $f_{1} \circ(g \circ \gamma) \circ f_{2}^{-1} \circ \gamma^{-1}$ can be filled in with paths, which gives a path in $L_{g} \tilde{M}$ connecting $f_{1}$ and $f_{2}$.

Now consider the evaluation maps (compare with Section 3)

$$
\Psi_{n}: L_{g} \tilde{M} \times \Delta^{n} \longrightarrow \tilde{M} \times \cdots \times \tilde{M}
$$

given by

$$
\Psi_{n}\left(f,\left(t_{1}, \cdots, t_{n}\right)\right):=\left(f(0), f\left(t_{1}\right), \cdots, f\left(t_{n}\right)\right) .
$$

On the chain level this leads to a chain complex which is rather similar to the cocyclic cobar complex:

Definition 9.2. Let $(C, \Delta, d)$ be a coassociative DG coalgebra over a field $k$. Suppose $G$ is a discrete group and $C$ admits a $k[G]$-action, which commutes with $\Delta$. Let $\Omega(C)$ be the cobar construction of $C$. Fix $g \in G$. Define an operator

$$
b_{g}: C \otimes \Omega(C) \longrightarrow C \otimes \Omega(C)
$$

by

$$
\begin{aligned}
& b_{g}\left(x \otimes\left[a_{1}|\cdots| a_{n}\right]\right) \\
& :=d x \otimes\left[a_{1}|\cdots| a_{n}\right]-\sum_{i}(-1)^{|x|+\left|\left[a_{1}|\cdots| a_{i-1}\right]\right|} x \otimes\left[a_{1}|\cdots| d a_{i}|\cdots| a_{n}\right] \\
& -\sum_{i} \sum_{\left(a_{i}\right)}(-1)^{|x|+\left|\left[a_{1}|\cdots| a_{i-1} \mid a_{i}^{\prime}\right]\right|} x \otimes\left[a_{1}|\cdots| a_{i}^{\prime}\left|a_{i}^{\prime \prime}\right| \cdots \mid a_{n}\right] \\
& +\sum_{(x)}(-1)^{\left|x^{\prime}\right|}\left(x^{\prime} \otimes\left[x^{\prime \prime}\left|a_{1}\right| \cdots \mid a_{n}\right]-(-1)^{\left(\left|x^{\prime \prime}\right|-1\right)\left(\left|\left[a_{1}|\cdots| a_{n}\right]\right|\right)} x^{\prime} \otimes\left[\left.a_{1}|\cdots| a_{n}\right|_{*} x^{\prime \prime}\right]\right) ;
\end{aligned}
$$

then $b_{g}^{2}=0$.

Consider the direct sum of $\left(C \otimes \Omega(C), b_{g}\right)$ indexed by $G$, and denote it by

$$
\left(C \otimes \Omega(C) \otimes k[G], \tilde{b}=\sum_{g \in G} b_{g}\right) .
$$

Define a $k[G]$-action on it by

$$
\begin{aligned}
& (k[G], C \otimes \Omega(C) \otimes k[G]) \quad \longrightarrow \quad C \otimes \Omega(C) \otimes k[G], \\
& \left(h, x \otimes\left[a_{1}|\cdots| a_{n}\right] \otimes g\right) \quad \longmapsto \quad h_{*} x \otimes\left[h_{*} a_{1}|\cdots| h_{*} a_{n}\right] \otimes h g h^{-1} .
\end{aligned}
$$

Moreover, define an operator $\tilde{B}$ on $C \otimes \Omega(C) \otimes k[G]$ as follows:

$$
\begin{aligned}
\tilde{B}: \quad C \otimes \Omega(C) \otimes k[G] & \longrightarrow C \otimes \Omega(C) \otimes k[G], \\
x \otimes\left[a_{1}|\cdots| a_{n}\right] \otimes g & \longmapsto \sum_{i}(-1)^{\epsilon} \varepsilon(x) a_{i} \otimes\left[a_{i+1}|\cdots| a_{n}\left|g_{*} a_{1}\right| \cdots \mid g_{*} a_{i-1}\right] \otimes g,
\end{aligned}
$$

where $\epsilon=\left|\left[a_{1}|\cdots| a_{i-1}\right]\right|\left|\left[a_{i}|\cdots| a_{n}\right]\right|$. The following lemma now holds by a direct calculation (where the reduced chain complex is used):

Lemma 9.3. Let $\left(C \otimes \Omega(C) \otimes k[G], b_{g}, \tilde{B}\right)$ be as above. Then:

(a) $\tilde{B}^{2}=0$ and $b_{g} \tilde{B}+\tilde{B} b_{g}=i d-g_{*}$.

(b) $\tilde{B}$ commutes with the $k[G]$-action. 
With this lemma, we may consider the $G$-equivariant complex

$$
(C \otimes \Omega(C) \otimes k[G]) / G=(C \otimes \Omega(C) \otimes k[G]) \otimes_{k[G]} k,
$$

where $\tilde{b}$ and $\tilde{B}$ descend to $b$ and $B$, with $b^{2}=0, B^{2}=0$ and $b B+B b=0$.

All the above definitions can be generalized to the complete case. Namely, for a complete DG coalgebra $C$ with a group $G$-action, we may consider the complete tensor product of $C$ with its complete cobar construction,

$$
(C \hat{\otimes} \hat{\Omega}(C) \otimes k[G], \tilde{b}, \tilde{B}),
$$

where $\tilde{b}$ and $\tilde{B}$ are the extensions of the usual boundary operators $\tilde{b}$ and $\tilde{B}$ to the completion. Also we may consider the $G$-equivariant complex

$$
(C \hat{\otimes} \hat{\Omega}(C) \otimes k[G] / G, b, B) .
$$

Therefore, for the DG open Frobenius-like algebra $\left(A_{\mathrm{c}}(\tilde{M}), C(\tilde{M}), \iota\right)$ on $\tilde{M}, A_{\mathrm{c}}(\tilde{M})$ $\hat{\otimes} \hat{\Omega}(C(\tilde{M})) \otimes g$ and $C(\tilde{M}) \hat{\otimes} \hat{\Omega}(C(\tilde{M})) \otimes g$ model the chain complex of $L_{g} \tilde{M}$, and by Lemma 9.1, the $G$-equivariant complex $\left(A_{\mathrm{c}}(\tilde{M}) \hat{\otimes} \hat{\Omega}(C(\tilde{M})) \otimes k[G]\right) / G$ and $(C(\tilde{M}) \hat{\otimes}$ $\hat{\Omega}(C(\tilde{M})) \otimes k[G]) / G$ model the chain complex of the free loop space $L M$.

To simplify the notation we write $A_{\mathrm{c}}(\tilde{M}) \hat{\otimes} \hat{\Omega}(C(\tilde{M})) \otimes g$ as $C_{*}\left(L_{g} \tilde{M}\right)$, and $\left(A_{\mathrm{c}}(\tilde{M}) \hat{\otimes} \hat{\Omega}(C(\tilde{M})) \otimes \mathbb{Q}[G]\right) / G$ as $C_{*}^{G}\left(\amalg L_{g} \tilde{M}\right)$, for short.

The loop product $\bullet$ of Chas-Sullivan is modeled as follows:

Definition 9.4 (Loop product). Let $\left(A_{\mathrm{c}}(\tilde{M}), C(\tilde{M}), \iota\right)$ be the DG Frobenius-like algebra of $\tilde{M}$. Define a binary operator $\tilde{\bullet}$ on $C_{*}\left(\amalg L_{g} \tilde{M}\right)$ as follows: for any

$$
\alpha=x \otimes\left[a_{1}|\cdots| a_{n}\right] \otimes g \in C_{*}\left(L_{g} \tilde{M}\right)
$$

and

$$
\beta=y \otimes\left[b_{1}|\cdots| b_{m}\right] \otimes h \in C_{*}\left(L_{h} \tilde{M}\right),
$$

let

$$
\alpha \tilde{\bullet} \beta:=(-1)^{(|y|+1)\left|\left[a_{1}|\cdots| a_{n}\right]\right|} x \cdot g_{*}^{-1} y \otimes\left[a_{1}|\cdots| a_{n}\left|b_{1}\right| \cdots \mid b_{m}\right] \otimes g h .
$$

On the $G$-equivariant chain complex $C_{*}^{G}\left(\amalg L_{g} \tilde{M}\right)$, define a binary operator • as follows: for $[\alpha],[\beta] \in C_{*}^{G}\left(\amalg L_{g} \tilde{M}\right)$,

$$
[\alpha] \bullet[\beta]:=\left[\alpha \tilde{\bullet} \sum_{g \in G} g_{*} \beta\right] .
$$

Remark 9.5. In [26, Section 3], Lupercio-Uribe-Xicotencatl gave a very similar construction as in Definition 9.4. Therefore, our algebraic construction of string topology here can be generalized to the orbifold case, too. The author is very grateful to the referee for pointing this out to him.

Lemma 9.6. The operator $\bullet$ does not depend on the choice of the representatives and is well defined. Moreover, it commutes with the boundary operator b.

Proof. The fact that $\bullet$ commutes with $b$ follows from a direct computation (compare with Definition 4.1 in the simply connected case). To show that $\bullet$ does not depend on the choice of representatives, take arbitrary $h, k \in G$,

$$
\begin{aligned}
{\left[h_{*} \alpha\right] \bullet\left[k_{*} \beta\right]=\left[h_{*} \alpha \tilde{\bullet} \sum_{g \in G} g_{*} k_{*} \beta\right] } & =\left[h_{*} \alpha \tilde{\bullet} \sum_{g \in G} g_{*} \beta\right]=\left[h_{*} \alpha \tilde{\bullet} \sum_{g \in G} h_{*} g_{*} \beta\right] \\
& =\left[h_{*}\left(\alpha \bullet \sum_{g \in G} g_{*} \beta\right)\right]=[\alpha] \bullet[\beta] .
\end{aligned}
$$


Also since $\mathbb{Q}[G]$ acts on $C_{*}\left(\coprod L_{g} \tilde{M}\right)$ freely and properly, and the differential forms are compactly supported, $\bullet$ is well defined.

Therefore we obtain a graded algebra on the homology of $H_{*}\left(C_{*}^{G}\left(\amalg L_{g} \tilde{M}\right), b\right)$. As in the simply connected case, such an algebra exactly models the loop product.

Definition 9.7 (* operator and the loop bracket). Let $\left(A_{\mathrm{c}}(\tilde{M}), C(\tilde{M}), \iota\right)$ be the DG open Frobenius-like algebra of $\tilde{M}$. Define a binary operator $\tilde{*}$ on $C_{*}\left(\amalg L_{g} \tilde{M}\right)$ as follows: for any

$$
\alpha=x \otimes\left[a_{1}|\cdots| a_{n}\right] \otimes g \in C_{*}\left(L_{g} \tilde{M}\right)
$$

and

$$
\beta=y \otimes\left[b_{1}|\cdots| b_{m}\right] \otimes h \in C_{*}\left(L_{h} \tilde{M}\right),
$$

let

$$
\begin{aligned}
\alpha \tilde{*} \beta:= & \sum_{i}(-1)^{|y|+(|y|-1)\left|\left[a_{i+1}|\cdots| a_{n}\right]\right|} \varepsilon\left(a_{i} y\right) x \\
& \otimes\left[a_{1}|\cdots| a_{i-1}\left|b_{1}\right| \cdots\left|b_{m}\right| h_{*} a_{i+1}|\cdots| h_{*} a_{n}\right] \otimes g h .
\end{aligned}
$$

On the $G$-equivariant chain complex $C_{*}^{G}\left(\amalg L_{g} \tilde{M}\right)$, define a binary operator * as follows: for $[\alpha],[\beta] \in C_{*}^{G}\left(\amalg L_{g} \tilde{M}\right)$,

$$
[\alpha] *[\beta]:=\left[\alpha \tilde{*} \sum_{g \in G} g_{*} \beta\right] .
$$

Lemma 9.8 (Gerstenhaber algebra of the free loop space). Let $M$ and $\tilde{M}$ be as above.

(1) $\operatorname{On} C_{*}\left(\amalg L_{g} \tilde{M}\right)$,

$$
b(\alpha \tilde{*} \beta)=b \alpha \tilde{*} \beta+(-1)^{|\alpha|+1} \alpha \tilde{*} b \beta+(-1)^{|\alpha|}\left(\alpha \tilde{\bullet} \beta-(-1)^{|\alpha||\beta|} h_{*}\left(h_{*}^{-1} \beta \tilde{\bullet} \alpha\right)\right) .
$$

(2) $O n C_{*}^{G}\left(\amalg L_{g} \tilde{M}\right)$, the operator $*$ does not depend on the choice of the representatives and hence is well defined. Moreover,

$$
b(\alpha * \beta)=b \alpha * \beta+(-1)^{|\alpha|+1} \alpha * b \beta+(-1)^{|\alpha|}\left(\alpha \bullet \beta-(-1)^{|\alpha||\beta|} \beta \bullet \alpha\right),
$$

which means that $\bullet$ is graded commutative on the homology $\mathbb{H}_{*}^{G}\left(\amalg L_{g} \tilde{M}\right)$.

(3) The commutator of $*$ forms a degree-one Lie algebra, which is compatible with $\bullet$, making

$$
\left(\mathbb{H}_{*}^{G}\left(\coprod L_{g} \tilde{M}\right), \bullet,\{,\}\right)
$$

a Gerstenhaber algebra.

Proof. These results follow from a direct computation.

Theorem 9.9 (Batalin-Vilkovisky algebra). Let $M$ be a smooth manifold and $\tilde{M}$ be its universal covering. The homology

$$
\left(\mathbb{H}_{*}^{G}\left(\coprod L_{g} \tilde{M}\right), \bullet, B\right)
$$

forms a Batalin-Vilkovisky algebra, which coincides with the one given by [6]. 
Proof. As in Definitions 9.4 and 9.7 the homotopy operator defined in Lemma 7.3 can be applied here, which implies the structure of a Batalin-Vilkovisky algebra.

To show that the Batalin-Vilkovisky algebra coincides with that of string topology, one may first identify the Batalin-Vilkovisky algebra of string topology of a nonsimply connected manifold with the one from its universal covering (cf. Lemma9.1). This is purely geometric and has been carried out by Lupercio-Uribe-Xicotencatl in 26. Namely, one may view $M$ as the orbifold $\tilde{M} / \pi_{1}(M)$. Then the BatalinVilkovisky algebra on $M$ constructed by Chas-Sullivan coincides with the one on $\tilde{M} / \pi_{1}(M)$ viewed as an orbifold (cf. [26, Section 5]). The algebraic construction of the Batalin-Vilkovisky parallel to [26] is exactly given above.

The construction of the gravity algebra on the equivariant homology is similar and is left to the interested reader.

\section{Appendix: Proof of Lemmas [5.8 and 7.3}

Proof of Lemma 5.8, (1) comes immediately from the definitions of $\bullet$ and $*$. We prove (2). In fact, up to sign,

$$
\begin{aligned}
& \gamma *(\alpha \bullet \beta)-(\gamma * \alpha) \bullet \beta-\alpha \bullet(\gamma * \beta) \\
= & \sum_{i} \varepsilon\left(c_{i} x y\right) z \otimes\left[c_{1}|\cdots| c_{i-1}\left|a_{1}\right| \cdots\left|a_{n}\right| b_{1}|\cdots| b_{m}\left|c_{i+1}\right| \cdots \mid c_{l}\right] \\
+ & \sum_{i} \varepsilon\left(c_{i} x\right) y z \otimes\left[c_{1}|\cdots| c_{i-1}\left|a_{1}\right| \cdots\left|a_{n}\right| c_{i+1}|\cdots| c_{l}\left|b_{1}\right| \cdots \mid b_{m}\right] \\
+ & \sum_{i} \varepsilon\left(c_{i} y\right) x z \otimes\left[a_{1}|\cdots| a_{n}\left|c_{1}\right| \cdots\left|c_{i-1}\right| b_{1}|\cdots| b_{m}\left|c_{i+1}\right| \cdots \mid c_{l}\right],
\end{aligned}
$$

while

$$
\begin{aligned}
& b \circ h(\alpha, \beta, \gamma) \\
& \text { (a4) } \quad=\sum_{i<j} \varepsilon\left(c_{i} x\right) \varepsilon\left(c_{j} y\right) d z \otimes\left[c_{1}|\cdots| c_{i-1}\left|a_{1}\right| \cdots\left|a_{n}\right| c_{i+1}|\cdots| c_{j-1}\left|b_{1}\right| \cdots\left|b_{m}\right| c_{j+1}|\cdots| c_{l}\right] \\
& (\mathrm{a} 5) \quad+\sum_{i<j, r} \varepsilon\left(c_{i} x\right) \varepsilon\left(c_{j} y\right) z \otimes\left[c_{1}|\cdots| c_{i-1}\left|a_{1}\right| \cdots\left|a_{n}\right| \cdots\left|d c_{r}\right| \cdots\left|c_{j-1}\right| b_{1}|\cdots| b_{m}|\cdots| c_{l}\right] \\
& \text { (a6) } \quad+\sum_{i<j, r} \varepsilon\left(c_{i} x\right) \varepsilon\left(c_{j} y\right) z \otimes\left[c_{1}|\cdots| c_{i-1}\left|a_{1}\right| \cdots\left|a_{n}\right| \cdots\left|c_{r}^{\prime}\right| c_{r}^{\prime \prime}|\cdots| c_{j-1}\left|b_{1}\right| \cdots\left|b_{m}\right| \cdots \mid c_{l}\right] \\
& \text { (a7) } \quad+\sum_{i<j, p} \varepsilon\left(c_{i} x\right) \varepsilon\left(c_{j} y\right) z \otimes\left[c_{1}|\cdots| c_{i-1}\left|a_{1}\right| \cdots\left|d a_{p}\right| \cdots\left|a_{n}\right| \cdots\left|c_{j-1}\right| b_{1}|\cdots| b_{m}|\cdots| c_{l}\right] \\
& \text { (a8) } \quad+\sum_{i<j, p} \varepsilon\left(c_{i} x\right) \varepsilon\left(c_{j} y\right) z \otimes\left[c_{1}|\cdots| c_{i-1}\left|a_{1}\right| \cdots\left|a_{p}^{\prime}\right| a_{p}^{\prime \prime}|\cdots| a_{n}|\cdots| c_{j-1}\left|b_{1}\right| \cdots\left|b_{m}\right| \cdots \mid c_{l}\right] \\
& \text { (a9) } \quad+\sum_{i<j, q} \varepsilon\left(c_{i} x\right) \varepsilon\left(c_{j} y\right) z \otimes\left[c_{1}|\cdots| c_{i-1}\left|a_{1}\right| \cdots\left|a_{n}\right| \cdots\left|c_{j-1}\right| b_{1}|\cdots| d b_{q}|\cdots| b_{m}|\cdots| c_{l}\right] \\
& (\mathrm{a} 10)+\sum_{i<j, q} \varepsilon\left(c_{i} x\right) \varepsilon\left(c_{j} y\right) z \otimes\left[c_{1}|\cdots| c_{i-1}\left|a_{1}\right| \cdots\left|a_{n}\right| \cdots\left|c_{j-1}\right| b_{1}|\cdots| b_{q}^{\prime}\left|b_{q}^{\prime \prime}\right| \cdots\left|b_{m}\right| \cdots \mid c_{l}\right] \\
& (\mathrm{a} 11)+\sum_{i<j} \varepsilon\left(c_{i} x\right) \varepsilon\left(c_{j} y\right) z^{\prime} \otimes\left[z^{\prime \prime}\left|c_{1}\right| \cdots\left|c_{i-1}\right| a_{1}|\cdots| a_{n}|\cdots| c_{j-1}\left|b_{1}\right| \cdots\left|b_{m}\right| \cdots \mid c_{l}\right] \\
& (\mathrm{a} 12)+\sum_{i<j} \varepsilon\left(c_{i} x\right) \varepsilon\left(c_{j} y\right) z^{\prime} \otimes\left[c_{1}|\cdots| c_{i-1}\left|a_{1}\right| \cdots\left|a_{n}\right| \cdots\left|c_{j-1}\right| b_{1}|\cdots| b_{m}|\cdots| c_{l} \mid z^{\prime \prime}\right]
\end{aligned}
$$


and

$h(b \alpha, \beta, \gamma)$

$(\mathrm{a} 13)=\sum_{i<j} \varepsilon\left(c_{i} d x\right) \varepsilon\left(c_{j} y\right) z \otimes\left[c_{1}|\cdots| c_{i-1}\left|a_{1}\right| \cdots\left|a_{n}\right| c_{i+1}|\cdots| c_{j-1}\left|b_{1}\right| \cdots\left|b_{m}\right| c_{j+1}|\cdots| c_{l}\right]$

$(\mathrm{a} 14)+\sum_{i<j, p} \varepsilon\left(c_{i} x\right) \varepsilon\left(c_{j} y\right) z \otimes\left[c_{1}|\cdots| c_{i-1}\left|a_{1}\right| \cdots\left|d a_{p}\right| \cdots\left|a_{n}\right| \cdots\left|c_{j-1}\right| b_{1}|\cdots| b_{m}|\cdots| c_{l}\right]$

$(\mathrm{a} 15)+\sum_{i<j, p} \varepsilon\left(c_{i} x\right) \varepsilon\left(c_{j} y\right) z \otimes\left[c_{1}|\cdots| c_{i-1}\left|a_{1}\right| \cdots\left|a_{p}^{\prime}\right| a_{p}^{\prime \prime}|\cdots| a_{n}|\cdots| c_{j-1}\left|b_{1}\right| \cdots\left|b_{m}\right| \cdots \mid c_{l}\right]$

$(\mathrm{a} 16)+\sum_{i<j} \varepsilon\left(c_{i} x^{\prime}\right) \varepsilon\left(c_{j} y\right) z \otimes\left[c_{1}|\cdots| c_{i-1}\left|x^{\prime \prime}\right| a_{1}|\cdots| a_{n}|\cdots| c_{j-1}\left|b_{1}\right| \cdots\left|b_{m}\right| \cdots \mid c_{l}\right]$

$(\mathrm{a} 17)+\sum_{i<j} \varepsilon\left(c_{i} x^{\prime}\right) \varepsilon\left(c_{j} y\right) z \otimes\left[c_{1}|\cdots| c_{i-1}\left|a_{1}\right| \cdots\left|a_{n}\right| x^{\prime \prime}|\cdots| c_{j-1}\left|b_{1}\right| \cdots\left|b_{m}\right| \cdots \mid c_{l}\right]$,

and

$h(\alpha, b \beta, \gamma)$

$(\mathrm{a} 18)=\sum_{i<j} \varepsilon\left(c_{i} x\right) \varepsilon\left(c_{j} d y\right) z \otimes\left[c_{1}|\cdots| c_{i-1}\left|a_{1}\right| \cdots\left|a_{n}\right| c_{i+1}|\cdots| c_{j-1}\left|b_{1}\right| \cdots\left|b_{m}\right| c_{j+1}|\cdots| c_{l}\right]$

$(\mathrm{a} 19)+\sum_{i<j, q} \varepsilon\left(c_{i} x\right) \varepsilon\left(c_{j} y\right) z \otimes\left[c_{1}|\cdots| c_{i-1}\left|a_{1}\right| \cdots\left|a_{n}\right| \cdots\left|c_{j-1}\right| b_{1}|\cdots| d b_{q}|\cdots| b_{m}|\cdots| c_{l}\right]$

$(\mathrm{a} 20)+\sum_{i<j, q} \varepsilon\left(c_{i} x\right) \varepsilon\left(c_{j} y\right) z \otimes\left[c_{1}|\cdots| c_{i-1}\left|a_{1}\right| \cdots\left|a_{n}\right| \cdots\left|c_{j-1}\right| b_{1}|\cdots| b_{q}^{\prime}\left|b_{q}^{\prime \prime}\right| \cdots\left|b_{m}\right| \cdots \mid c_{l}\right]$

$(\mathrm{a} 21)+\sum_{i<j} \varepsilon\left(c_{i} x\right) \varepsilon\left(c_{j} y^{\prime}\right) z \otimes\left[c_{1}|\cdots| c_{i-1}\left|a_{1}\right| \cdots\left|a_{n}\right| \cdots\left|c_{j-1}\right| y^{\prime \prime}\left|b_{1}\right| \cdots\left|b_{m}\right| \cdots \mid c_{l}\right]$

$(\mathrm{a} 22)+\sum_{i<j} \varepsilon\left(c_{i} x\right) \varepsilon\left(c_{j} y^{\prime}\right) z \otimes\left[c_{1}|\cdots| c_{i-1}\left|a_{1}\right| \cdots\left|a_{n}\right| \cdots\left|c_{j-1}\right| b_{1}|\cdots| b_{m}\left|y^{\prime \prime}\right| \cdots \mid c_{l}\right]$,

and one has similar terms for $h(\alpha, \beta, b \gamma)$. A straightforward check shows that all terms cancel except for (a1) $+(a 2)+(a 3)$. Thus (2) is proved.

Proof of Lemma 7.3. First note that $A \hat{\otimes} \hat{\Omega}(C)$ embeds in $C \hat{\otimes} \hat{\Omega}(C)$, so the operator $B$ is well defined. For $\alpha=x \otimes\left[a_{1}|\cdots| a_{n}\right], \beta=y \otimes\left[b_{1}|\cdots| b_{m}\right] \in A \hat{\otimes} \hat{\Omega}(C)$, define

$$
\phi(\alpha, \beta):=\sum_{i<j} \varepsilon(x) \varepsilon\left(a_{j} y\right) a_{i} \otimes\left[a_{i+1}|\cdots| a_{j-1}\left|b_{1}\right| \cdots\left|b_{m}\right| a_{j+1}|\cdots| a_{n}\left|a_{1}\right| \cdots \mid a_{i-1}\right]
$$

and

$$
\psi(\alpha, \beta):=\sum_{k<l} \varepsilon(y) \varepsilon\left(b_{l} x\right) b_{k} \otimes\left[b_{k+1}|\cdots| b_{l-1}\left|a_{1}\right| \cdots\left|a_{n}\right| b_{l+1}|\cdots| b_{m}\left|b_{1}\right| \cdots \mid b_{k-1}\right],
$$

and let $h=\phi+\psi$. We will show that $h$ thus defined satisfies (7.2). Since $A \hat{\otimes} \hat{\Omega}(C)$ and $C \hat{\otimes} \hat{\Omega}(C)$ have the same homology, (7.1) follows from (7.2).

In fact, $\{\alpha, \beta\}-(-1)^{|\alpha|} B(\alpha \bullet \beta)-(-1)^{(|\beta|+1)(|\alpha|+1)} \beta \bullet B(\alpha)+\alpha \bullet B(\beta)$ contains two parts:

$$
\begin{aligned}
& \sum_{i} \varepsilon(x y) a_{i} \otimes\left[a_{i+1}|\cdots| a_{n}\left|b_{1}\right| \cdots\left|b_{m}\right| a_{1}|\cdots| a_{i-1}\right] \\
+ & \sum_{i} \varepsilon\left(a_{i} y\right) x \otimes\left[a_{1}|\cdots| a_{i-1}\left|b_{1}\right| \cdots\left|b_{m}\right| a_{i+1}|\cdots| a_{n}\right] \\
+ & \sum_{i} \varepsilon(x) a_{i} y \otimes\left[b_{1}|\cdots| b_{m}\left|a_{i+1}\right| \cdots\left|a_{n}\right| a_{1}|\cdots| a_{i-1}\right]
\end{aligned}
$$


and

$$
\begin{aligned}
& \sum_{k} \varepsilon(x y) b_{k} \otimes\left[b_{k+1}|\cdots| b_{m}\left|a_{1}\right| \cdots\left|a_{n}\right| b_{1}|\cdots| b_{k-1}\right] \\
+ & \sum_{k} \varepsilon\left(b_{k} x\right) y \otimes\left[b_{1}|\cdots| b_{k-1}\left|a_{1}\right| \cdots\left|a_{n}\right| b_{k+1}|\cdots| b_{m}\right] \\
+ & \sum_{k} \varepsilon(y) b_{k} x \otimes\left[a_{1}|\cdots| a_{n}\left|b_{k+1}\right| \cdots\left|b_{m}\right| b_{1}|\cdots| b_{k-1}\right] .
\end{aligned}
$$

while

$$
b \phi(\alpha, \beta)
$$

$$
=\sum_{i<j} \varepsilon(x) \varepsilon\left(a_{j} y\right) d a_{i} \otimes\left[a_{i+1}|\cdots| a_{j-1}\left|b_{1}\right| \cdots\left|b_{m}\right| a_{j+1}|\cdots| a_{n}\left|a_{1}\right| \cdots \mid a_{i-1}\right]
$$

$$
+\sum_{i<j, p} \varepsilon(x) \varepsilon\left(a_{j} y\right) a_{i} \otimes\left[a_{i+1}|\cdots| d a_{p}|\cdots| a_{j-1}\left|b_{1}\right| \cdots\left|b_{m}\right| a_{j+1}|\cdots| a_{n}\left|a_{1}\right| \cdots \mid a_{i-1}\right]
$$

$$
+\sum_{i<j, p} \varepsilon(x) \varepsilon\left(a_{j} y\right) a_{i} \otimes\left[a_{i+1}|\cdots| a_{p}^{\prime}\left|a_{p}^{\prime \prime}\right| \cdots\left|a_{j-1}\right| b_{1}|\cdots| b_{m}\left|a_{j+1}\right| \cdots\left|a_{n}\right| a_{1}|\cdots| a_{i-1}\right]
$$

$$
+\sum_{i<j, q} \varepsilon(x) \varepsilon\left(a_{j} y\right) a_{i} \otimes\left[a_{i+1}|\cdots| a_{j-1}\left|b_{1}\right| \cdots\left|d b_{q}\right| \cdots\left|b_{m}\right| a_{j+1}|\cdots| a_{n}\left|a_{1}\right| \cdots \mid a_{i-1}\right]
$$

$$
+\sum_{i<j, q} \varepsilon(x) \varepsilon\left(a_{j} y\right) a_{i} \otimes\left[a_{i+1}|\cdots| a_{j-1}\left|b_{1}\right| \cdots\left|b_{q}^{\prime}\right| b_{q}^{\prime \prime}|\cdots| b_{m}\left|a_{j+1}\right| \cdots\left|a_{n}\right| a_{1}|\cdots| a_{i-1}\right]
$$

$$
+\sum_{i<j} \varepsilon(x) \varepsilon\left(a_{j} y\right) a_{i}^{\prime} \otimes\left[a_{i}^{\prime \prime}\left|a_{i+1}\right| \cdots\left|a_{j-1}\right| b_{1}|\cdots| b_{m}\left|a_{j+1}\right| \cdots\left|a_{n}\right| a_{1}|\cdots| a_{i-1}\right]
$$

$$
+\sum_{i<j} \varepsilon(x) \varepsilon\left(a_{j} y\right) a_{i}^{\prime \prime} \otimes\left[a_{i+1}|\cdots| a_{j-1}\left|b_{1}\right| \cdots\left|b_{m}\right| a_{j+1}|\cdots| a_{n}\left|a_{1}\right| \cdots\left|a_{i-1}\right| a_{i}^{\prime}\right] .
$$

and

$$
\phi(b \alpha, \beta)
$$

$$
=\sum_{i<j, p} \varepsilon\left(a_{j} y\right) \varepsilon(x) a_{i} \otimes\left[a_{i+1}|\cdots| d a_{p}|\cdots| a_{j-1}\left|b_{1}\right| \cdots\left|b_{m}\right| a_{j+1}|\cdots| a_{n}\left|a_{1}\right| \cdots \mid a_{i-1}\right]
$$

$$
+\sum_{i<j, p} \varepsilon\left(a_{j} y\right) \varepsilon(x) a_{i} \otimes\left[a_{i+1}|\cdots| a_{p}^{\prime}\left|a_{p}^{\prime \prime}\right| \cdots\left|a_{j-1}\right| b_{1}|\cdots| b_{m}\left|a_{j+1}\right| \cdots\left|a_{n}\right| a_{1}|\cdots| a_{i-1}\right]
$$

$$
+\sum_{i<j} \varepsilon(x) \varepsilon\left(a_{j} y\right) d a_{i} \otimes\left[a_{i+1}|\cdots| a_{j-1}\left|b_{1}\right| \cdots\left|b_{m}\right| a_{j+1}|\cdots| a_{n}\left|a_{1}\right| \cdots \mid a_{i-1}\right]
$$

$$
+\sum_{i<j} \varepsilon(x) \varepsilon\left(d a_{j} y\right) a_{i} \otimes\left[a_{i+1}|\cdots| a_{j-1}\left|b_{1}\right| \cdots\left|b_{m}\right| a_{j+1}|\cdots| a_{n}\left|a_{1}\right| \cdots \mid a_{i-1}\right]
$$


(a40)

$$
+\sum_{i<j} \varepsilon(x) \varepsilon\left(a_{j}^{\prime} y\right) a_{i} \otimes\left[a_{i+1}|\cdots| a_{j-1}\left|b_{1}\right| \cdots\left|b_{m}\right| a_{j}^{\prime \prime}\left|a_{j+1}\right| \cdots\left|a_{n}\right| a_{1}|\cdots| a_{i-1}\right]
$$

(a41)

$$
+\sum_{i<j} \varepsilon(x) \varepsilon\left(a_{j}^{\prime \prime} y\right) a_{i} \otimes\left[a_{i+1}|\cdots| a_{j-1}\left|a_{j}^{\prime}\right| b_{1}|\cdots| b_{m}\left|a_{j+1}\right| \cdots\left|a_{n}\right| a_{1}|\cdots| a_{i-1}\right]
$$

$$
+\sum_{i<j} \varepsilon(x) \varepsilon\left(a_{j} y\right) a_{i}^{\prime} \otimes\left[a_{i}^{\prime \prime}|\cdots| a_{j-1}\left|b_{1}\right| \cdots\left|b_{m}\right| a_{j+1}|\cdots| a_{n}\left|a_{1}\right| \cdots \mid a_{i-1}\right]
$$

(a43)

$$
+\sum_{i<j} \varepsilon(x) \varepsilon\left(a_{j} y\right) a_{i}^{\prime \prime} \otimes\left[a_{i+1}|\cdots| a_{j-1}\left|b_{1}\right| \cdots\left|b_{m}\right| a_{j+1}|\cdots| a_{n}\left|a_{1}\right| \cdots\left|a_{i-1}\right| a_{i}^{\prime}\right]
$$

(a44)

$$
+\sum_{i} \varepsilon(x) \varepsilon\left(a_{i}^{\prime \prime} y\right) a_{i}^{\prime} \otimes\left[b_{1}|\cdots| b_{m}\left|a_{i+1}\right| \cdots\left|a_{n}\right| a_{1}|\cdots| a_{i-1}\right]
$$

$$
+\sum_{i} \varepsilon\left(x^{\prime}\right) \varepsilon\left(a_{i} y\right) x^{\prime \prime} \otimes\left[a_{1}|\cdots| a_{i-1}\left|b_{1}\right| \cdots\left|b_{m}\right| a_{i+1}|\cdots| a_{n}\right]
$$

$$
+\sum_{i} \varepsilon\left(x^{\prime}\right) \varepsilon\left(x^{\prime \prime} y\right) a_{i} \otimes\left[a_{i+1}|\cdots| a_{n}\left|b_{1}\right| \cdots\left|b_{m}\right| a_{1}|\cdots| a_{i-1}\right]
$$

and

$$
\begin{aligned}
& (\alpha, b \beta) \\
(\mathrm{a} 47) & =\sum_{i<j} \varepsilon(x) \varepsilon\left(a_{j} d y\right) a_{i} \otimes\left[a_{i+1}|\cdots| a_{j-1}\left|b_{1}\right| \cdots\left|b_{m}\right| a_{j+1}|\cdots| a_{n}\left|a_{1}\right| \cdots \mid a_{i-1}\right] \\
(\mathrm{a} 48) & +\sum_{i<j, q} \varepsilon(x) \varepsilon\left(a_{j} y\right) a_{i} \otimes\left[a_{i+1}|\cdots| a_{j-1}\left|b_{1}\right| \cdots\left|d b_{q}\right| \cdots\left|b_{m}\right| a_{j+1}|\cdots| a_{n}\left|a_{1}\right| \cdots \mid a_{i-1}\right] \\
(\mathrm{a} 49) & +\sum_{i<j, q} \varepsilon(x) \varepsilon\left(a_{j} y\right) a_{i} \otimes\left[a_{i+1}|\cdots| a_{j-1}\left|b_{1}\right| \cdots\left|b_{q}^{\prime}\right| b_{q}^{\prime \prime}|\cdots| b_{m}\left|a_{j+1}\right| \cdots\left|a_{n}\right| a_{1}|\cdots| a_{i-1}\right] \\
(\mathrm{a} 50) & +\sum_{i<j} \varepsilon(x) \varepsilon\left(a_{j} y^{\prime}\right) a_{i} \otimes\left[a_{i+1}|\cdots| a_{j-1}\left|y^{\prime \prime}\right| b_{1}|\cdots| b_{m}\left|a_{j+1}\right| \cdots\left|a_{n}\right| a_{1}|\cdots| a_{i-1}\right] \\
(\mathrm{a} 51) & +\sum_{i<j} \varepsilon(x) \varepsilon\left(a_{j} y^{\prime}\right) a_{i} \otimes\left[a_{i+1}|\cdots| a_{j-1}\left|b_{1}\right| \cdots\left|b_{m}\right| y^{\prime \prime}\left|a_{j+1}\right| \cdots\left|a_{n}\right| a_{1}|\cdots| a_{i-1}\right] .
\end{aligned}
$$

Note that (a36) and (a30) are identical, so are (a38) and (a29), a39) and (a47), (a37) and (a31), (a42) and (a34), (a43) and (a35), (a40) and (a51), (a41) and (a50), a32) and (a48), and (a33) and (a49); therefore the remaining terms of $b \phi(\alpha, \beta)-\phi(b \alpha, \beta)-\phi(\alpha, b \beta)$ are exactly (a23) $+(\mathrm{a} 24)+(\mathrm{a} 25)$.

Similarly $b \psi(\alpha, \beta)-\psi(b \alpha, \beta)-\psi(\alpha, b \beta)$ is equal to (a26) + (a27) + a28).

\section{ACKNOWLEDGMENTS}

The author would like to thank Dennis Sullivan, Yongbin Ruan, James McClure, John McCleary, John Terilla, Thomas Tradler, Scott Wilson and Dezhen Xu for many helpful conversations. He also thanks the referee for very carefully reading the paper and pointing out numerous errors and imprecise statements in the first draft of the paper. 


\section{REFERENCES}

[1] Adams, J.F., On the cobar construction. Proc. Nat. Acad. Sci. U.S.A. 42 (1956), 409-412. MR.0079266 (18:59c)

[2] Adem, A. and Ruan, Y., Twisted orbifold K-theory. Comm. Math. Phys. 237 (2003), no. 3, 533-556. MR:1993337(2004e:19004)

[3] Bousfield, A.K., On the homology spectral sequence of a cosimplicial space. Amer. J. Math. 109 (1987), no. 2, 361-394. MR882428 (88j:55017)

[4] Brown, E., Twisted tensor products, I. Ann. of Math. (2), Vol. 69, No. 1, January, 1959, pp. 223-246. MR0105687 (21:4423)

[5] Cenkl, B. and Porter, R., de Rham theorem with cubical forms. Pacific J. Math. 112 (1984), no. 1, 35-48. MR739139 (85j:55009)

[6] Chas, M. and Sullivan, D., String topology, arXiv:math-GT/9911159.

[7] Chas, M. and Sullivan, D., Closed string operators in topology leading to Lie bialgebras and higher string algebra, in The legacy of Niels Henrik Abel, 771-784, Springer, Berlin, 2004. MR2077595 (2005f:55007)

[8] Chen, K.-T., Iterated path integrals. Bull. Amer. Math. Soc. 83 (1977), 831-879. MR0454968 $(56: 13210)$

[9] Cohen, R., Hess, K. and Voronov, A.A. String topology and cyclic homology. Advanced Courses in Mathematics. CRM Barcelona. Birkhäuser Verlag, Basel, 2006. MR2251006 (2007f:55001)

[10] Cohen, R. and Jones, J.D.S., A homotopy theoretic realization of string topology. Math. Ann. 324, 773-798(2002). MR1942249 (2004c:55019)

[11] Cohen, R., Klein, J. and Sullivan, D., The homotopy invariance of the string topology loop product and string bracket, Journal of Topology 1 (2008), no. 2, 391-408. MR 2399136 (2009h:55004)

[12] Connes, A., Noncommutative differential geometry. Inst. Hautes Études Sci. Publ. Math. No. 62 (1985), 257-360. MR823176 (87i:58162)

[13] Costello, K., Topological conformal field theories and Calabi-Yau categories. Adv. Math. 210 (2007), no. 1, 165-214. MR2298823 (2008f:14071)

[14] Félix, Y. and Thomas, J.-C., Rational BV-algebra in String Topology, Bull. Soc. Math. France 136 (2008), no. 2, 311-327. MR2415345 (2009c:55015)

[15] Félix, Y., Thomas, J.-C., and Vigué-Poirrier, M., Rational string topology, J. Eur. Math. Soc. (JEMS) 9 (2007), no. 1, 123-156. MR2283106 (2007k:55009)

[16] Gerstenhaber, M., The cohomology structure of an associative ring. Ann. of Math. (2) 78 (1963), 267-288. MR0161898(28:5102)

[17] Getzler, E., Batalin-Vilkovisky algebras and two-dimensional topological field theories. Comm. Math. Phys. 159 (1994), no. 2, 265-285. MR1256989 (95h:81099)

[18] Getzler, E., Two-dimensional topological gravity and equivariant cohomology. Comm. Math. Phys. 163 (1994), 473-489. MR1284793 (95h:81100)

[19] Getzler, E., Jones, J.D.S. and Petrack, S., Differential forms on loop spaces and the cyclic bar complex. Topology 30 (1991), 339-371. MR.1113683 (92i:58179)

[20] Godin, V., Higher string topology operations, arxiv:0711.4859.

[21] Hamilton, A. and Lazarev, A., Homotopy algebras and noncommutative geometry, arxiv:math.QA/0403340.

[22] Jones, J.D.S., Cyclic homology and equivariant homology. Invent. Math. 87 (1987), 403-423. MR.870737 (88f:18016)

[23] Keller, B., Hochschild cohomology and derived Picard groups, J. of Pure and Applied Algebra 190 (2004), 177-196. MR2043327 (2004m:16012)

[24] Kaufmann, R., A proof of a cyclic version of Deligne's conjecture via cacti. Math. Res. Lett. 15 (2008), no. 5, 901-921. MR2443991(2009i:18006)

[25] Loday, J.-L., Cyclic homology. Second edition. Grundlehren der Mathematischen Wissenschaften, 301. Springer-Verlag, Berlin, 1998. MR1600246 (98h:16014)

[26] Lupercio, E., Uribe, B. and Xicotencatl, M.A., Orbifold string topology. Geom. Topol. 12 (2008), no. 4, 2203-2247. MR2431019 (2009k:55016)

[27] McCleary, J., Homotopy theory and closed geodesics, in Homotopy theory and related topics (Kinosaki, 1988), 86-94, Lecture Notes in Math., 1418, Springer, Berlin, 1990. MR.1048178 (91e:57060) 
[28] McCleary, J., A user's guide to spectral sequences. Second edition. Cambridge Studies in Advanced Mathematics, 58. Cambridge University Press, Cambridge, 2001. MR 1793722 (2002c:55027)

[29] McClure, J., On the chain-level intersection pairing for PL manifolds, math.QA/0410450, and a talk given at Morelia, Mexico, 2006. Geom. Topol. 10 (2006), 1391-1424. MR.2255502 (2007f:57048)

[30] Menichi, L., Batalin-Vilkovisky algebras and cyclic cohomology of Hopf algebras. K-Theory 32 (2004), no. 3, 231-251. MR2114167 (2006c:16018)

[31] Menichi, L., Batalin-Vilkovisky algebra structures on Hochschild cohomology, Bull. Soc. Math. France 137 (2009), no. 2, 277-295. MR2543477 (2011b:16034)

[32] Merkulov, A., De Rham model for string topology. Int. Math. Res. Not. 2004, no. 55, 29552981. MR 2099178 (2005g:57055)

[33] Quillen, D., Rational homotopy theory. Ann. of Math. (2) 90 (1969), 205-295. MR0258031 (41:2678)

[34] Rector, D.L., Steenrod operations in the Eilenberg-Moore spectral sequence. Comment. Math. Helv. 45 (1970), 540-552. MR0278310 (43:4040)

[35] Sullivan, D., Open and closed string field theory interpreted in classical algebraic topology. Topology, geometry and quantum field theory, 344-357, London Math. Soc. Lecture Note Ser., 308, Cambridge Univ. Press, Cambridge, 2004. MR2079379 (2005g:81289)

[36] Sullivan, D., String topology: background and present state. Current Developments in Mathematics 2005 41-88, Int. Press, Somerville, MA, 2007. MR2459297 (2010c:55007)

[37] Tradler, T., Two BV-structures identified: The Hochschild cohomology and the homology of the free loop space. CUNY Ph.D. Thesis, 2002.

[38] Voronov, A.A., Homotopy Gerstenhaber algebras, in Conférence Moshé Flato 1999, Vol. II, Math. Phys. Stud. 22 (2000), 307-331. MR:1805923 (2002d:55009)

[39] Voronov, A.A., Notes on universal algebra. Graphs and patterns in mathematics and theoretical physics, 81-103, Proc. Sympos. Pure Math., 73, Amer. Math. Soc., Providence, RI, 2005. MR 2131012 (2005k:18017)

[40] Voronov, A.A. and Gerstenhaber, M., Higher-order operations on the Hochschild complex. (Russian) Funktsional. Anal. i Prilozhen. 29 (1995), no. 1, 1-6, 96; translation in Funct. Anal. Appl. 29 (1995), no. 1, 1-5. MR1328534 (96g:18006)

[41] Westerland, C., Equivariant operads, string topology, and Tate cohomology. Math. Ann. 340 (2008), no. 1, 97-142. MR2349769 (2008k:55014)

[42] Whitehead, J.H.C., On $C^{1}$-complexes. Ann. of Math. (2) 41 (1940), 809-824. MR.0002545 $(2: 73 \mathrm{~d})$

[43] Wilson, S., Partial algebras over operads of complexes and applications, arXiv:math. $\mathrm{AT} / 0410405$.

School of Mathematics, Sichuan University, Chengdu 610064, People's Republic of China - And - Department of Mathematics, University of Michigan, 530 Church Street, Ann Arbor, Michigan 48104

E-mail address: xch@umich.edu 